\title{
Vibrational cooling, heating, and instability in molecular conducting junctions: Full counting statistics analysis
}

\author{
Lena Simine and Dvira Segal \\ Chemical Physics Theory Group, Department of Chemistry, \\ University of Toronto, 80 Saint George St. Toronto, Ontario, Canada M5S $3 H 6$
}

(Dated: November 5, 2018)

\begin{abstract}
We study current-induced vibrational cooling, heating, and instability in a donor-acceptor rectifying molecular junction using a full counting statistics approach. In our model, electron-hole pair excitations are coupled to a given molecular vibrational mode which is either harmonic or highly anharmonic. This mode may be further coupled to a dissipative thermal environment. Adopting a master equation approach, we confirm the charge and heat exchange fluctuation theorem in the steady-state limit, for both harmonic and anharmonic models. Using simple analytical expressions, we calculate the charge current and several measures for the mode effective temperature. At low bias, we observe the effect of bias-induced cooling of the vibrational mode. At higher bias, the mode effective temperature is higher than the environmental temperature, yet the junction is stable. Beyond that, once the vibrational mode (bias-induced) excitation rate overcomes its relaxation rate, instability occurs. We identify regimes of instability as a function of voltage bias and coupling to an additional phononic thermal bath. Interestingly, we observe a reentrant behavior where an unstable junction can properly behave at a high enough bias. The mechanism for this behavior is discussed.
\end{abstract}

PACS numbers:

\section{INTRODUCTION}

Can molecules serve as reliable components in electronic circuits? A major obstacle in realizing molecularbased electronic devices is junction heating and breakdown, the result of vibrational excitation by the electron current [1-10]. This situation generally occurs once the bias voltage exceeds typical molecular vibrational frequencies and the electronic levels are situated within the bias window. If energy dissipation from the conducting object to its environment (metals, solvent) is not efficient, the molecular conductor experiences significant heating, ultimately leading to junction breakdown. A related question, the possibility for a nonequilibrium induced cooling of the junction has been the topic of recent experimental and theoretical studies [4, 11-13].

In this paper, we study the problem of bias-induced molecular cooling, heating, and (potential) junction breakdown due to vibrational instabilities, using the Donor (D)-Acceptor (A) Aviram-Ratner electronic rectifier setup [14], see Fig. 1. By coupling electronic transitions within the junction to a particular internal molecular vibrational mode, significant molecular heating can take place once the donor level is lifted above the acceptor level, as the excess electronic energy is used to excite the vibrational mode. This process may ultimately lead to junction instabilities and breakdown [16]. The model can also demonstrate current-induced cooling at low bias, when tuning the junction's parameters.

Within this simple system, several issues are of fundamental and practical interest. First, one would like to understand the role of mode anharmonicity in the transport process and in the heating or cooling behavior. Second, the molecular vibration under investigation, the one controlling junction stability, can be assumed to be well isolated from other modes. Alternatively, this mode may be coupled to other phonons, allowing for energy damping to a larger environment. These two situations should result in distinctive cooling or heating behaviors. These issues will be explored here. Other relevant challenges which are not considered here are the possibility to selectively excite vibrational modes in the molecule, using voltage bias [17], or more generally, to drive molecular motion or trigger chemical dynamics [18].
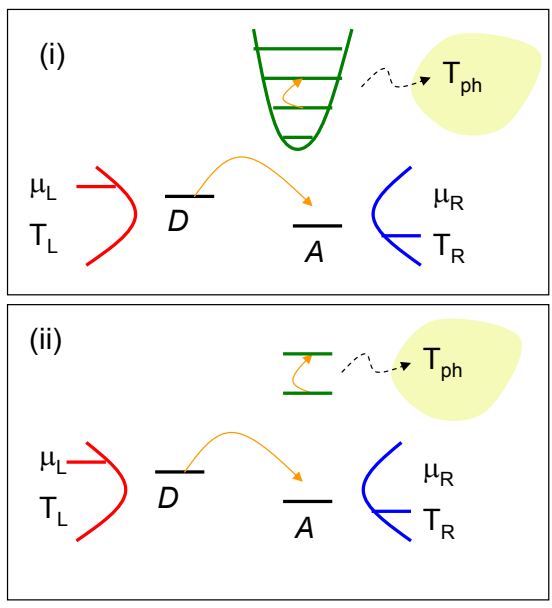

FIG. 1: Schemes of the two models considered in this work. A biased donor-acceptor electronic junction is coupled to either (i) a harmonic molecular mode, or (ii) an anharmonic mode, represented by a two-state system. In both cases the molecular mode may further relax its energy to a phononic thermal reservoir, maintained at the temperature $T_{p h}$. This coupling is represented by a dashed arrow.

Using a full counting statistics (FCS) approach, our analysis further contributes to the institution of fluc- 
tuation relations in open many-body quantum systems. Fluctuation theorems (FT) for entropy production quantifies the probability of negative entropy generation, measuring "second law violation" $[19,20]$. Such "anomalous" processes are relevant at the nanoscale. While originally demonstrated in classical systems [21], recent experimental efforts are dedicated to explore their validity within quantum systems [22]. From the theoretical side, the extension of the work and heat FT to the quantum domain has recently attracted significant attention [23, 24]. Specifically, a quantum exchange FT, for the transfer of charge and energy between two reservoirs maintained at different chemical potentials and temperatures, has been derived in Ref. [25] using projective measurements, and in Ref. [23] based on the unraveling of the quantum master equation. It is of interest to test these relations in particular cases, e.g., for systems strongly coupled to multiple reservoirs, when the reservoirs cooperatively affect the subsystem [26], including nonmarkovian reservoirs [27-29], and in models showing coupled charge and energy transfer processes, yet the respective fluxes are not tightly coupled. The system investigated here corresponds to the latter case.

Different flavors of the phonon-assisted-tunneling model have been analyzed in the literature [9]. Among the various techniques adopted we list solution of the dynamics as a scattering problem [30], extension of the basic nonequilibrium Green's function formalism to include molecular vibrations [31], or the use of master equation approaches [32]. In this paper, we exploit the latter method, and present a full-counting statistics of the system, allowing for the exploration of charge current, energy current and noise processes at the same footing. Further, we analytically obtain the cumulant generating function (CGF) of the model, allowing for the verification of the steady-state charge-energy fluctuation theorem in this many-body quantum system.

The objectives of this work are therefore twofold: (i) to analyze a simple model that can elucidate cooling, heating and instability mechanisms in molecular rectifiers, specifically, to understand the roles of mode anharmonicity and additional damping routes, and (ii) to establish the steady-state entropy production fluctuation theorem within a nonequilibrium quantum model, transferring charge and energy between the reservoirs in a cooperative manner. Recent studies have analyzed the role of electron-vibration interaction on the full counting statistics (FCS) within different approaches [28, 3336]. Complementing these efforts, our treatment offers an analytic structure for the CGF, allowing for a clear inspection of the microscopic processes involved.

The plan of the paper is as follows. In Sec. II we introduce the D-A molecular rectifier and its two flavors, either including a harmonic or an anharmonic internal vibration. In Sec. III the anharmonic model is analyzed within a FCS approach, demonstrating cooling, heating and instability dynamics at different parameter regions. The case with an additional phonon bath is considered in
Appendix A. Sec. IV explores the harmonic mode model. Sec. V concludes.

\section{MODEL}

Our model includes a biased molecular electronic junction and a selected internal vibrational mode which is coupled to an electronic transition in the junction. This mode possibly interacts with other (reservoir) phonons, an extension presented in Appendix A. For a schematic representation, see Fig. 1. Generally, this model allows one to investigate the exchange of electronic energy with molecular (vibrational) heating. The total Hamiltonian is given by the following terms,

$$
H=H_{M}+H_{L}+H_{R}+H_{c}+H_{v i b}+H_{I} .
$$

The first term, $H_{M}$, stands for the molecular electronic part including two electronic states

$$
H_{M}=\epsilon_{d} c_{d}^{\dagger} c_{d}+\epsilon_{a} c_{a}^{\dagger} c_{a} .
$$

Here, $c_{d / a}^{\dagger}\left(c_{d / a}\right)$ is a fermionic creation (annihilation) operator of an electron on the donor or acceptor sites, of energies $\epsilon_{d, a}$. The second and third terms in Eq. (1) describe the two metals, $H_{\nu}, \nu=L, R$, each including a collection of noninteracting electrons

$$
H_{L}=\sum_{l \in L} \epsilon_{l} c_{l}^{\dagger} c_{l} ; \quad H_{R}=\sum_{r \in R} \epsilon_{r} c_{r}^{\dagger} c_{r} .
$$

The hybridization of the donor state to the left $(L)$ bath, and similarly, the coupling of the acceptor site to the right $(R)$ metal, are incorporated into $H_{c}$,

$$
H_{c}=\sum_{l} v_{l}\left(c_{l}^{\dagger} c_{d}+c_{d}^{\dagger} c_{l}\right)+\sum_{r} v_{r}\left(c_{r}^{\dagger} c_{a}+c_{a}^{\dagger} c_{r}\right)
$$

The Hamiltonian further includes an internal molecular vibrational mode of frequency $\omega_{0}$. The mode displacement from equilibrium is coupled to an electron hopping in the system with an energy cost $\kappa$, resulting in heating and/or cooling effects,

$$
\begin{aligned}
H_{v i b} & =\omega_{0} b_{0}^{\dagger} b_{0} \\
H_{I} & =\kappa\left[c_{d}^{\dagger} c_{a}+c_{a}^{\dagger} c_{d}\right]\left(b_{0}^{\dagger}+b_{0}\right)
\end{aligned}
$$

Here, $b_{0}^{\dagger}\left(b_{0}\right)$ represents a bosonic creation (annihilation) operator. Note that in our construction the donor and acceptor sites are coupled to each other only through the interaction with the vibrational mode. We now diagonalize the electronic part of the Hamiltonian, $H_{e l}=$ $H_{M}+H_{L}+H_{R}+H_{c}$, to obtain, separately, the exact eigenstates for the $L$-half and $R$-half of $H_{e l}$,

$$
H_{e l}=\sum_{l} \epsilon_{l} a_{l}^{\dagger} a_{l}+\sum_{r} \epsilon_{r} a_{r}^{\dagger} a_{r} .
$$


Assuming that the reservoirs are dense, their new operators are assigned energies same as those before diagonalization. The donor and acceptor (new) energies are assumed to be placed within the band of continuous states, excluding the existence of bound states. The old operators are related to the exact eigenstates by [37]

$$
\begin{aligned}
c_{d} & =\sum_{l} \lambda_{l} a_{l}, & c_{l} & =\sum_{l^{\prime}} \eta_{l, l^{\prime}} a_{l^{\prime}} \\
c_{a} & =\sum_{r} \lambda_{r} a_{r}, & c_{r} & =\sum_{r^{\prime}} \eta_{r, r^{\prime}} a_{r^{\prime}},
\end{aligned}
$$

where the coefficients, e.g., for the $L$ set, are given by

$$
\begin{aligned}
\lambda_{l} & =\frac{v_{l}}{\epsilon_{l}-\epsilon_{d}-\sum_{l^{\prime}} \frac{v_{l^{\prime}}^{2}}{\epsilon_{l}-\epsilon_{l^{\prime}}+i \delta}} \\
\eta_{l, l^{\prime}} & =\delta_{l, l^{\prime}}-\frac{v_{l} \lambda_{l^{\prime}}}{\epsilon_{l}-\epsilon_{l^{\prime}}+i \delta} .
\end{aligned}
$$

Similar expressions hold for the $R$ set. It is easy to derive the following relation,

$$
\sum_{l^{\prime}} \frac{v_{l^{\prime}}^{2}}{\epsilon_{l}-\epsilon_{l^{\prime}}+i \delta}=P P \sum_{l^{\prime}} \frac{v_{l^{\prime}}^{2}}{\epsilon_{l}-\epsilon_{l^{\prime}}}-i \Gamma_{L}\left(\epsilon_{l}\right) / 2
$$

with the hybridization strength $\Gamma_{L}(\epsilon)=2 \pi \sum_{l} v_{l}^{2} \delta\left(\epsilon-\epsilon_{l}\right)$. The expectation values of the exact eigenstates are

$$
\left\langle a_{l}^{\dagger} a_{l^{\prime}}\right\rangle=\delta_{l, l^{\prime}} f_{L}\left(\epsilon_{l}\right), \quad\left\langle a_{r}^{\dagger} a_{r^{\prime}}\right\rangle=\delta_{r, r^{\prime}} f_{R}\left(\epsilon_{r}\right),
$$

where $f_{L}(\epsilon)=\left[\exp \left(\beta_{L}\left(\epsilon-\mu_{L}\right)\right)+1\right]^{-1}$ denotes the Fermi distribution function. An analogous expression holds for $f_{R}(\epsilon)$. The reservoirs temperatures are denoted by $1 / \beta_{\nu}$; the chemical potentials are $\mu_{\nu}$. With the new operators, the Hamiltonian (1) can be rewritten as

$$
\begin{aligned}
H_{H} & =\sum_{l} \epsilon_{l} a_{l}^{\dagger} a_{l}+\sum_{r} \epsilon_{r} a_{r}^{\dagger} a_{r}+\omega_{0} b_{0}^{\dagger} b_{0} \\
& +\kappa \sum_{l, r}\left[\lambda_{l}^{*} \lambda_{r} a_{l}^{\dagger} a_{r}+\lambda_{r}^{*} \lambda_{l} a_{r}^{\dagger} a_{l}\right]\left(b_{0}^{\dagger}+b_{0}\right) .
\end{aligned}
$$

In this form, the model generally describes the process of an electron-hole pair excitation by a molecular vibration. We denote it by $H_{H}$, to highlight the vibrational mode harmonicity. A simple version of the model is reached by replacing the harmonic mode by a two-state system (spin), using the Pauli matrices,

$$
\begin{aligned}
H_{A} & =\sum_{l} \epsilon_{l} a_{l}^{\dagger} a_{l}+\sum_{r} \epsilon_{r} a_{r}^{\dagger} a_{r}+\frac{\omega_{0}}{2} \sigma_{z} \\
& +\kappa \sum_{l, r}\left[\lambda_{l}^{*} \lambda_{r} a_{l}^{\dagger} a_{r}+\lambda_{r}^{*} \lambda_{l} a_{r}^{\dagger} a_{l}\right] \sigma_{x} .
\end{aligned}
$$

The truncated harmonic spectrum imitates an anharmonic mode, as only several (two in the present extreme case) states are bounded within the anharmonic potential [38]. We denote this Hamiltonian by $H_{A}$, to indicate on the anharmonicity of the molecular mode. The dynamics of this model should coincide with the behavior dictated by $H_{H}$, at low temperatures.

Charge and energy transfer dynamics in these models can be followed by studying electronic properties $[35,36,39]$. In contrast, here we explore the junction response to an applied voltage bias by studying the vibrational mode excitation and relaxation dynamics. The analysis of the two-state model, Eq. (12), therefore turns out to be simpler than the case when the vibrational mode has an infinite spectrum. In what follows, we derive in details the CGF for the anharmonic-mode case. Appendix A generalizes this calculation to include an additional dissipative thermal bath. We then extend these results and discuss the model conveyed by Eq. (11).
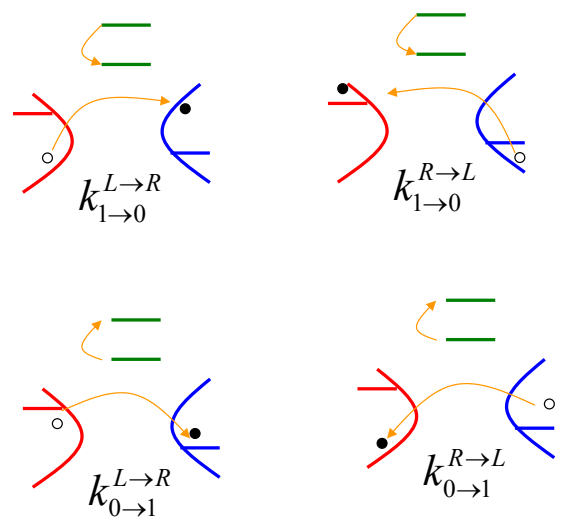

FIG. 2: Scheme of the vibrational mode excitation and relaxation processes. A full circle represents an electron transferred; a hollow circle depicts the hole that has been left behind.

\section{ANHARMONIC-MODE RECTIFIER}

\section{A. Impurity dynamics}

We explore the dynamics of an anharmonic mode, referred to as an "impurity", or a two-state-system (TLS), within an electronic rectifier, assuming a weak donoracceptor - mode interaction. We rewrite Eq. (12) as

$$
H_{A}=\frac{\omega_{0}}{2} \sigma_{z}+\sigma_{x} F_{e}+\sum_{l} \epsilon_{l} a_{l}^{\dagger} a_{l}+\sum_{r} \epsilon_{r} a_{r}^{\dagger} a_{r},
$$

by defining the electron-hole pair generation operator as

$$
F_{e}=\kappa \sum_{l, r}\left(\lambda_{l}^{*} \lambda_{r} a_{l}^{\dagger} a_{r}+\lambda_{r}^{*} \lambda_{l} a_{r}^{\dagger} a_{l}\right) .
$$

The Hamiltonian (13) can be transformed to the spinfermion model $[40,41]$ of zero energy spacing, using the unitary transformation

$$
U^{\dagger} \sigma_{z} U=\sigma_{x}, \quad U^{\dagger} \sigma_{x} U=\sigma_{z},
$$


with $U=\frac{1}{\sqrt{2}}\left(\sigma_{x}+\sigma_{z}\right)$. The transformed Hamiltonian $\tilde{H}_{A}=U^{\dagger} H_{A} U$ is given by

$$
\tilde{H}_{A}=\frac{\omega_{0}}{2} \sigma_{x}+\sigma_{z} F_{e}+\sum_{l} \epsilon_{l} a_{l}^{\dagger} a_{l}+\sum_{r} \epsilon_{r} a_{r}^{\dagger} a_{r} .
$$

In this form, the TLS dynamics can be simulated exactly using an influence-functional path integral approach [42].

Back to (13), we denote the TLS ground state and excited state by $|0\rangle$ and $|1\rangle$, with energies 0 and $\omega_{0}$, respectively. We express the Pauli operators by these states, $\sigma_{z}=|1\rangle\langle 1|-| 0\rangle\left\langle 0\left|, \sigma_{x}=\right| 1\right\rangle\langle 0|+| 0\rangle\langle 1|$. Next, using the quantum Liouville equation, we obtain kinetic-rate equations for the states population $p_{n}(n=0,1)[38,43]$. This standard derivation involves a second order perturbation theory treatment with respect to $\kappa$, the mode-molecule coupling parameter, followed by a Markov approximation. The resulting equation for the reduce density matrix $\rho_{S}$ take the simple form $(\hbar \equiv 1)$

$$
\begin{aligned}
\dot{\rho}_{S} & =-i\left[V(t), \rho_{S}(0)\right] \\
& -\int_{0}^{\infty} d \tau \operatorname{Tr}_{B}\left\{\left[V(t),\left[V(\tau), \rho_{S}(t) \rho_{L} \rho_{R}\right]\right]\right\}
\end{aligned}
$$

Here, $V=\sigma_{x} F_{e}$ represents the (mode-molecule) coupling term in Eq. (13). The operators are written in the interaction representation, $O(t)=e^{i\left(H_{A}-V\right) t} O e^{-i\left(H_{A}-V\right) t}$ and we trace over the electronic degrees of freedom. The reservoirs $\nu=L, R$ are maintained in a grand canonical state as $\rho_{\nu}=e^{-\beta_{\nu}\left(H_{\nu}-\mu_{\nu} N_{\nu}\right)} / Z_{\nu} ; Z_{\nu}$ is the partition function of the $\nu$ bath. Identifying the diagonal matrix elements as population, $p_{n}=\left[\rho_{S}\right]_{n, n}$, we obtain the kinetic equation

$$
\dot{p}_{1}=-k_{1 \rightarrow 0}^{e} p_{1}+k_{0 \rightarrow 1}^{e} p_{0}, \quad p_{1}+p_{0}=1 .
$$

In this model, the off-diagonal elements of the reduced density matrix are naturally decoupled from the population dynamics [44]. The excitation $\left(k_{0 \rightarrow 1}^{e}\right)$ and relaxation $\left(k_{1 \rightarrow 0}^{e}\right)$ rate constants are given by Fourier transforms of bath correlation functions

$$
\begin{aligned}
& k_{1 \rightarrow 0}^{e}=\int_{-\infty}^{\infty} e^{i \omega_{0} \tau}\left\langle F_{e}(\tau) F_{e}(0)\right\rangle d \tau \\
& k_{0 \rightarrow 1}^{e}=\int_{-\infty}^{\infty} e^{-i \omega_{0} \tau}\left\langle F_{e}(\tau) F_{e}(0)\right\rangle d \tau,
\end{aligned}
$$

enclosing electron-hole pair excitation processes,

$$
\begin{aligned}
\left\langle F_{e}(t) F_{e}(0)\right\rangle & =\kappa^{2} \operatorname{Tr}_{L} \operatorname{Tr}_{R}\left\{\sum_{l, l^{\prime}} \sum_{r, r^{\prime}} \rho_{L} \rho_{R}\right. \\
& \times\left[\lambda_{l}^{*} \lambda_{r} a_{l}^{\dagger}(t) a_{r}(t)+\lambda_{r}^{*} \lambda_{l} a_{r}^{\dagger}(t) a_{l}(t)\right] \\
& \left.\times\left[\lambda_{l^{\prime}}^{*} \lambda_{r^{\prime}} a_{l^{\prime}}^{\dagger}(0) a_{r^{\prime}}(0)+\lambda_{r^{\prime}}^{*} \lambda_{l^{\prime}} a_{r^{\prime}}^{\dagger}(0) a_{l^{\prime}}(0)\right]\right\} .
\end{aligned}
$$

The operators are given in the interaction representation, e.g., $a_{l}^{\dagger}(t)=e^{i H_{L} t} a_{l}^{\dagger} e^{-i H_{L} t}$. As we separately trace over the $L$ and $R$-baths' degrees of freedom, it can be shown that the rate constants can be decomposed into two contributions,

$$
k_{1 \rightarrow 0}^{e}=k_{1 \rightarrow 0}^{L \rightarrow R}+k_{1 \rightarrow 0}^{R \rightarrow L} ; \quad k_{0 \rightarrow 1}^{e}=k_{0 \rightarrow 1}^{L \rightarrow R}+k_{0 \rightarrow 1}^{R \rightarrow L},
$$

satisfying

$$
\begin{aligned}
k_{1 \rightarrow 0}^{L \rightarrow R} & =2 \pi \kappa^{2} \\
& \times \sum_{l, r}\left|\lambda_{l}\right|^{2}\left|\lambda_{r}\right|^{2} f_{L}\left(\epsilon_{l}\right)\left(1-f_{R}\left(\epsilon_{r}\right)\right) \delta\left(\omega_{0}+\epsilon_{l}-\epsilon_{r}\right) \\
k_{0 \rightarrow 1}^{L \rightarrow R} & =2 \pi \kappa^{2} \\
& \times \sum_{l, r}\left|\lambda_{l}\right|^{2}\left|\lambda_{r}\right|^{2} f_{L}\left(\epsilon_{l}\right)\left(1-f_{R}\left(\epsilon_{r}\right)\right) \delta\left(-\omega_{0}+\epsilon_{l}-\epsilon_{r}\right) .
\end{aligned}
$$

Similar relations hold for the right-to-left going excitations. The energy in the Fermi function $f_{\nu}(\epsilon)$ is measured with respect to the (equilibrium) Fermi energy, placed at $\left(\mu_{L}+\mu_{R}\right)$, and we assume that the bias is applied symmetrically, $\mu_{L}=-\mu_{R}$. The four rate constants describe distinct electron-hole excitation processes, depicted in Fig. 2. At forward bias, if we set the effective density of states (DOS) of the $L$ bath to lie higher in energy that the DOS of the right bath, we immediately note that the rate $k_{0 \rightarrow 1}^{L \rightarrow R}$ should dominate over $k_{1 \rightarrow 0}^{L \rightarrow R}$, potentially leading to "population inversion" of the vibrational mode. Utilizing electronic reservoirs with energy dependent DOS is thus the basic ingredient of the instability formation here, as we show below. For convenience, we define the spectral density for the $\nu$ bath as

$$
J_{\nu}(\epsilon)=2 \pi \kappa \sum_{j \in \nu}\left|\lambda_{j}\right|^{2} \delta\left(\epsilon_{j}-\epsilon\right) .
$$

Explicitly, using Eq. (8), we find that this function has a Lorentzian lineshape, and that it is centered around either the D or A level,

$$
\begin{aligned}
& J_{L}(\epsilon)=\kappa \frac{\Gamma_{L}(\epsilon)}{\left(\epsilon-\epsilon_{d}\right)^{2}+\Gamma_{L}(\epsilon)^{2} / 4} \\
& J_{R}(\epsilon)=\kappa \frac{\Gamma_{R}(\epsilon)}{\left(\epsilon-\epsilon_{a}\right)^{2}+\Gamma_{R}(\epsilon)^{2} / 4} .
\end{aligned}
$$

Using the spectral density function, we express the terms in Eq. (22) as integrals

$$
\begin{aligned}
& k_{1 \rightarrow 0}^{L \rightarrow R}=\frac{1}{2 \pi} \int_{-\infty}^{\infty} f_{L}(\epsilon)\left[1-f_{R}\left(\epsilon+\omega_{0}\right)\right] J_{L}(\epsilon) J_{R}\left(\epsilon+\omega_{0}\right) d \epsilon \\
& k_{1 \rightarrow 0}^{R \rightarrow L}=\frac{1}{2 \pi} \int_{-\infty}^{\infty} f_{R}(\epsilon)\left[1-f_{L}\left(\epsilon+\omega_{0}\right)\right] J_{R}(\epsilon) J_{L}\left(\omega_{0}+\epsilon\right) d \epsilon \\
& k_{0 \rightarrow 1}^{L \rightarrow R}=\frac{1}{2 \pi} \int_{-\infty}^{\infty} f_{L}(\epsilon)\left[1-f_{R}\left(\epsilon-\omega_{0}\right)\right] J_{L}(\epsilon) J_{R}\left(\epsilon-\omega_{0}\right) d \epsilon \\
& k_{0 \rightarrow 1}^{R \rightarrow L}=\frac{1}{2 \pi} \int_{-\infty}^{\infty} f_{R}(\epsilon)\left[1-f_{L}\left(\epsilon-\omega_{0}\right)\right] J_{R}(\epsilon) J_{L}\left(\epsilon-\omega_{0}\right) d \epsilon .
\end{aligned}
$$


The following relations hold $\left(\beta_{L}=\beta_{R}\right.$ and $\Delta \mu \equiv \mu_{L}-$ $\left.\mu_{R}\right)$,

$$
\frac{k_{1 \rightarrow 0}^{R \rightarrow L}}{k_{0 \rightarrow 1}^{L \rightarrow R}}=e^{-\beta \Delta \mu} e^{\beta \omega_{0}} ; \quad \frac{k_{1 \rightarrow 0}^{L \rightarrow R}}{k_{0 \rightarrow 1}^{R \rightarrow L}}=e^{\beta \Delta \mu} e^{\beta \omega_{0}} .
$$

In equilibrium, detailed bias is therefore maintained.

The dynamics conveyed by Eqs. (18)-(25) is nonseparable in terms of the two metals, in contrast to simple linear interaction cases [38]. In other words, the reservoirs cooperatively excite or damp energy from the impurity, thus their action is non-additive.

It should be noted that while we assume a weak interaction limit, between electron-hole pair generation and the vibrational mode, our scheme does not enforce weak metal-molecule coupling; this part is exactly diagonalized to yield the reservoirs spectral function, peaked about the $\mathrm{D}$ or $\mathrm{A}$ levels. If one where to force weak metalmolecule interaction, the spectral functions (24) would reduce to delta functions, $J_{L}(\epsilon)=2 \pi \kappa \delta\left(\epsilon-\epsilon_{d}\right)$ and $J_{R}(\epsilon)=2 \pi \kappa \delta\left(\epsilon-\epsilon_{a}\right)$, and the resulting rates would be evaluated at the donor and acceptor levels, e.g., $k_{1 \rightarrow 0}^{L \rightarrow R}=$ $2 \pi \kappa^{2} f_{L}\left(\epsilon_{d}\right)\left[1-f_{R}\left(\epsilon_{a}\right)\right] \delta\left(\epsilon_{d}-\epsilon_{a}+\omega_{0}\right)$. This also implies that charge and energy currents are not "tightly coupled" here, such that for each transferred electron not necessarily precisely one quanta of energy should be gained or drained at either contact. In this aspect, our study complements the work reported in [10]. There, using the small polaron transformation, the coupling of the molecular bridge to the leads is assumed to be weak, while its coupling to the vibrational mode can be made large.

\section{B. Resolved charge and energy equations}

We write here a closed expression for the cumulant generating function, following the approach developed in Refs. [26, 27]. It will allow us to obtain the current, its noise power, and to confirm the FTs in this system. We define $\mathcal{P}_{t}(n, N, \omega)$ as the probability that by the time $t$ the impurity (TLS) occupies the state $n, N$ electrons have been transferred from the $L$ metal to the $R$ side, and a net energy $\omega$ has been transferred, $L$ to $R$. Resolving Eq. (18) to its charge and energy components, we find that this probability satisfies the following equation of motion $[26,27]$,

$$
\begin{aligned}
\dot{\mathcal{P}}_{t}(1, N, \omega) & =-\mathcal{P}_{t}(1, N, \omega) k_{1 \rightarrow 0}^{e} \\
& +\int_{-\infty}^{\infty} \mathcal{P}_{t}\left(0, N-1, \omega-\epsilon+\omega_{0}\right) f_{L}(\epsilon)\left[1-f_{R}\left(\epsilon-\omega_{0}\right)\right] J_{L}(\epsilon) J_{R}\left(\epsilon-\omega_{0}\right) d \epsilon \\
& +\int_{-\infty}^{\infty} \mathcal{P}_{t}(0, N+1, \omega+\epsilon) f_{R}(\epsilon)\left[1-f_{L}\left(\epsilon-\omega_{0}\right)\right] J_{R}(\epsilon) J_{L}\left(\epsilon-\omega_{0}\right) d \epsilon \\
\dot{\mathcal{P}}_{t}(0, N, \omega) & =-\mathcal{P}_{t}(0, N, \omega) k_{0 \rightarrow 1}^{e} \\
& +\int_{-\infty}^{\infty} \mathcal{P}_{t}\left(1, N-1, \omega-\epsilon-\omega_{0}\right) f_{L}(\epsilon)\left[1-f_{R}\left(\epsilon+\omega_{0}\right)\right] J_{L}(\epsilon) J_{R}\left(\epsilon+\omega_{0}\right) d \epsilon \\
& +\int_{-\infty}^{\infty} \mathcal{P}_{t}(1, N+1, \omega+\epsilon) f_{R}(\epsilon)\left[1-f_{L}\left(\epsilon+\omega_{0}\right)\right] J_{R}(\epsilon) J_{L}\left(\epsilon+\omega_{0}\right) d \epsilon
\end{aligned}
$$

One could reason this rate equation as follows. In the first equation, the term $\mathcal{P}_{t}(1, N, \omega) k_{1 \rightarrow 0}^{e}$ stands for the decay rate of $\mathcal{P}_{t}(1, N, \omega)$. The second line describes a process where by the time $t$ the TLS occupies the ground state, $N-1$ excess electrons have arrived at the $R$ terminal, and an overall of $\omega-\epsilon+\omega_{0}$ energy has been absorbed at the $R$ bath. At the time $t$ an electron-hole pair excitation generates an electron at the $R$ bath, leaving a hole at the $L$ metal. This charge transfer process is accompanied by an electronic energy transmission at the amount of $\epsilon-\omega_{0}$ : An electron leaving the $L$ bath has a total energy $\epsilon$, however only $\epsilon-\omega_{0}$ is gained by the $R$ bath. The rest, at the amount of $\omega_{0}$, is gained by the vibrational mode. A similar reasoning can explain other terms in Eq. (27). For convenience, the factor $(2 \pi)^{-1}$ in Eq. (25) has been absorbed into the definition of $J_{\nu}(\omega)$.

We Fourier transform the above system of equations with respect to both charge and energy, to obtain the characteristic function $\mathcal{Z}(\chi, \eta, t)$. It depends on the energy counting field $\eta$ and the charge counting field $\chi$,

$$
|\mathcal{Z}(\chi, \eta, t)\rangle \equiv\left(\begin{array}{l}
\sum_{N=-\infty}^{\infty} e^{i N \chi} \int_{-\infty}^{\infty} \mathcal{P}_{t}(0, N, \omega) e^{i \omega \eta} d \omega \\
\sum_{N=-\infty}^{\infty} e^{i N \chi} \int_{-\infty}^{\infty} \mathcal{P}_{t}(1, N, \omega) e^{i \omega \eta} d \omega
\end{array}\right)
$$

It satisfies the differential equation

$$
\frac{d|\mathcal{Z}(\chi, \eta, t)\rangle}{d t}=-\hat{\mathcal{W}}(\chi, \eta)|\mathcal{Z}(\chi, \eta, t)\rangle
$$


where the matrix $\hat{\mathcal{W}}$ contains the following elements

$$
\begin{aligned}
& \hat{\mathcal{W}}(\chi, \eta)= \\
& \left(\begin{array}{cc}
k_{0 \rightarrow 1}^{L \rightarrow R}+k_{0 \rightarrow 1}^{R \rightarrow L} & -e^{i \chi} F_{1}^{-}(\eta)-e^{-i \chi} F_{2}^{+}(\eta) \\
-e^{i \chi} F_{1}^{+}(\eta)-e^{-i \chi} F_{2}^{-}(\eta) & k_{1 \rightarrow 0}^{L \rightarrow R}+k_{1 \rightarrow 0}^{R \rightarrow L}
\end{array}\right)
\end{aligned}
$$

Here,

$$
\begin{aligned}
& F_{1}^{ \pm}(\eta)= \\
& \int_{-\infty}^{\infty} e^{i \epsilon \eta} f_{L}\left(\epsilon \pm \omega_{0}\right)\left[1-f_{R}(\epsilon)\right] J_{L}\left(\epsilon \pm \omega_{0}\right) J_{R}(\epsilon) d \epsilon \\
& F_{2}^{ \pm}(\eta)= \\
& \int_{-\infty}^{\infty} e^{-i \epsilon \eta}\left[1-f_{L}\left(\epsilon \pm \omega_{0}\right)\right] f_{R}(\epsilon) J_{L}\left(\epsilon \pm \omega_{0}\right) J_{R}(\epsilon) d \epsilon
\end{aligned}
$$

The cumulant generating function is formally defined as

$$
\begin{aligned}
& G(\chi, \eta)= \\
& \lim _{t \rightarrow \infty} \frac{1}{t} \ln \sum_{N=-\infty}^{\infty} e^{i N \chi} \int_{-\infty}^{\infty} \mathcal{P}_{t}(N, \omega) e^{i \omega \eta} d \omega,
\end{aligned}
$$

where we introduced the short notation $\mathcal{P}_{t}(N, \omega)=$ $\mathcal{P}_{t}(0, N, \omega)+\mathcal{P}_{t}(1, N, \omega)$, that is the probability to transfer by the time $t, N$ electrons and an energy $\omega$ from left to right, irrespective of the state of the TLS. The charge and heat currents can be readily derived, by taking the first derivative of the CGF with respect to either $\eta$ or $\chi$,

$$
\begin{aligned}
& \left\langle I_{e}\right\rangle \equiv \frac{\langle N\rangle_{t}}{t}=\left.\frac{d G(\chi, \eta)}{d(i \chi)}\right|_{\chi=0, \eta=0} \\
& \left\langle I_{q}\right\rangle \equiv \frac{\langle\omega\rangle_{t}}{t}=\left.\frac{d G(\chi, \eta)}{d(i \eta)}\right|_{\chi=0, \eta=0}
\end{aligned}
$$

The quantity $\langle\omega\rangle_{t}$ denotes the total energy $\omega$ transferred from $L$ to $R$ by the (infinitely long) time $t ;\langle N\rangle_{t}$ similarly counts the particles (electrons) transferred in the same direction, by that time. The zero frequency noise current power density can be similarly obtained,

$$
\begin{aligned}
& \left\langle S_{e}\right\rangle \equiv \frac{\left\langle N^{2}\right\rangle_{t}-\langle N\rangle_{t}^{2}}{t}=\left.\frac{d^{2} G(\chi, \eta)}{d(i \chi)^{2}}\right|_{\chi=0, \eta=0} \\
& \left\langle S_{q}\right\rangle \equiv \frac{\left\langle\omega^{2}\right\rangle_{t}-\langle\omega\rangle_{t}^{2}}{t}=\left.\frac{d^{2} G(\chi, \eta)}{d(i \eta)^{2}}\right|_{\chi=0, \eta=0}
\end{aligned}
$$

The CGF can be expressed in terms of $|\mathcal{Z}\rangle$ as

$$
G(\chi, \eta)=\lim _{t \rightarrow \infty} \frac{1}{t} \ln \langle I \mid \mathcal{Z}(\chi, \eta, t)\rangle,
$$

with $\langle I|=\langle 11|$, a left vector of unity. It is practically given by the negative of the smallest eigenvalue of the matrix $\hat{\mathcal{W}}$,

$$
\begin{aligned}
G(\chi, \eta) & =-\frac{w_{1,1}+w_{2,2}}{2} \\
& +\frac{\sqrt{\left(w_{1,1}-w_{2,2}\right)^{2}+4 w_{1,2}(\chi, \eta) w_{2,1}(\chi, \eta)}}{2} .
\end{aligned}
$$

$w_{i, j}$ are the matrix elements of $\hat{\mathcal{W}}$, see Eq. (30).

\section{Fluctuation theorem}

We confirm next the following symmetry

$$
G(\chi, \eta)=G\left(-\chi+i\left(\beta_{L} \mu_{L}-\beta_{R} \mu_{R}\right),-\eta+i \Delta \beta\right),
$$

with $\Delta \beta=\beta_{R}-\beta_{L}$. In order to prove this, we focus on the product $D(\chi, \eta) \equiv w_{1,2}(\chi, \eta) w_{2,1}(\chi, \eta)$ in Eq. (36),

$$
\begin{aligned}
D(\chi, \eta) & =\left[e^{i \chi} F_{1}^{-}(\eta)+e^{-i \chi} F_{2}^{+}(\eta)\right] \\
& \times\left[e^{i \chi} F_{1}^{+}(\eta)+e^{-i \chi} F_{2}^{-}(\eta)\right] .
\end{aligned}
$$

Under the transformation $\chi \rightarrow-\chi+i\left(\beta_{L} \mu_{L}-\beta_{R} \mu_{R}\right)$ and $\eta \rightarrow-\eta+i \Delta \beta$, using the relation $f_{\nu}(\epsilon)=[1-$ $\left.f_{\nu}(\epsilon)\right] e^{-\beta_{\nu}\left(\epsilon-\mu_{\nu}\right)}$, we find that

$$
\begin{aligned}
e^{i \chi} F_{1}^{-}(\eta) & \rightarrow e^{-i \chi} e^{-\beta_{L} \mu_{L}+\beta_{R} \mu_{R}} \int_{-\infty}^{\infty} d \epsilon e^{-i \epsilon \eta} e^{-\Delta \beta \epsilon}\left[1-f_{L}\left(\epsilon-\omega_{0}\right)\right] e^{-\beta_{L}\left(\epsilon-\omega_{0}-\mu_{L}\right)} f_{R}(\epsilon) e^{\beta_{R}\left(\epsilon-\mu_{R}\right)} J_{L}\left(\epsilon-\omega_{0}\right) J_{R}(\epsilon) \\
& =e^{-i \chi} e^{\beta_{L} \omega_{0}} F_{2}^{-}(\eta) \\
e^{-i \chi} F_{2}^{+}(\eta) & \rightarrow e^{i \chi} e^{\beta_{L} \mu_{L}-\beta_{R} \mu_{R}} \int_{-\infty}^{\infty} d \epsilon e^{i \epsilon \eta} e^{\Delta \beta \epsilon}\left[1-f_{R}(\epsilon)\right] e^{-\beta_{R}\left(\epsilon-\mu_{R}\right)} f_{L}\left(\epsilon+\omega_{0}\right) e^{\beta_{L}\left(\epsilon+\omega_{0}-\mu_{L}\right)} J_{L}\left(\epsilon+\omega_{0}\right) J_{R}(\epsilon) \\
& =e^{i \chi} e^{\beta_{L} \omega_{0}} F_{1}^{+}(\eta) .
\end{aligned}
$$

Similarly, one could show that

$$
\begin{gathered}
e^{i \chi} F_{1}^{+}(\eta) \rightarrow e^{-i \chi e^{-\beta_{L} \omega_{0}}} F_{2}^{+}(\eta) \\
e^{-i \chi} F_{2}^{-}(\eta) \rightarrow e^{i \chi e^{-\beta_{L} \omega_{0}}} F_{1}^{-}(\eta) .
\end{gathered}
$$

The extra factors $e^{ \pm \beta_{L} \omega_{0}}$ cancel, and we recover the symmetry

$$
D(\chi, \eta)=D\left(-\chi+i\left(\beta_{L} \mu_{L}-\beta_{R} \mu_{R}\right),-\eta+i \Delta \beta\right),
$$


confirming Eq. (37). We can now demonstrate the validity of a fluctuation relation for this non-equilibrium system. The probability to transfer the energy $\omega$ by the long time $t$, from $L$ to $R$, is given by the inverse Fourier transform of Eq. (32),

$\mathcal{P}_{t}(N, \omega) \sim \frac{1}{2 \pi} \sum_{-\infty}^{\infty} e^{-i N \chi} \int_{-\infty}^{\infty} C(\chi, \eta) e^{G(\chi, \eta) t} e^{-i \omega \eta} d \eta$

with $\lim _{t \rightarrow \infty}[\ln C(\chi, \eta)] / t=0$. Similarly, the quantity $\mathcal{P}_{t}(-N,-\omega)$ represents the probability that $N$ charged particles and an energy $\omega$ have been transmitted in the opposite direction, right to left, up to time $t$. Based on the symmetry Eq. (37), one can show that [23]

$$
\lim _{t \rightarrow \infty} \frac{1}{t} \ln \frac{\mathcal{P}_{t}(N, \omega)}{\mathcal{P}_{t}(-N,-\omega)}=\frac{\omega \Delta \beta+N\left(\beta_{L} \mu_{L}-\beta_{R} \mu_{R}\right)}{t}
$$

which is often written in a compact form as

$$
\frac{\mathcal{P}_{t}(N, \omega)}{\mathcal{P}_{t}(-N,-\omega)}=e^{\omega \Delta \beta+N\left(\beta_{L} \mu_{L}-\beta_{R} \mu_{R}\right)} .
$$

This expression goes beyond standard metal-molecule weak-coupling schemes as the energy and charge transfer and not tightly coupled, and the energy $\omega$ can take continuous values, unlike Refs. [39, 46, 47].

It should be noted that the above derivation has assumed charge and energy conservation between the two reservoirs. The full particle-energy counting statistics, without such an assumption, would begin with the probability distribution $\mathcal{P}_{t}\left(n, N_{L}, N_{R}, \omega_{L}, \omega_{R}\right)$, to find the system at time $t$ in the spin state $n=0,1$, with $N_{\nu}$ electrons and $\omega_{\nu}$ excess energy accumulated at the $\nu$ bath. One can readily write an equation of motion for this function, analogous to Eq. (27), to be Fourier transformed using four counting fields,

$$
\begin{aligned}
& \mathcal{P}_{t}\left(n, \chi_{L}, \chi_{R}, \eta_{L}, \eta_{R}\right)=\sum_{N_{L}} e^{i N_{L} \chi_{L}} \sum_{N_{R}} e^{i N_{R} \chi_{R}} \\
& \times \int_{-\infty}^{\infty} e^{i \omega_{L} \eta_{L}} d \omega_{L} \int_{-\infty}^{\infty} e^{i \omega_{R} \eta_{R}} \mathcal{P}_{t}\left(n, N_{L}, N_{R}, \omega_{L}, \omega_{R}\right) d \omega_{R}
\end{aligned}
$$

This quantity satisfies an equation of motion that is analogous to Eq. (29). It can be readily proved that the negative of the smallest eigenvalue of the corresponding matrix $\hat{\mathcal{W}}\left(\chi_{L}, \chi_{R}, \eta_{L}, \eta_{R}\right)$ obeys the symmetry

$$
\begin{aligned}
& G\left(\chi_{L}, \chi_{R}, \eta_{L}, \eta_{R}\right)= \\
& G\left(-\chi_{L}+i \beta_{L} \mu_{L},-\chi_{R}+i \beta_{R} \mu_{R},-\eta_{L}+i \beta_{L},-\eta_{R}+i \beta_{R}\right),
\end{aligned}
$$

which can be translated into the FT for the probability itself,

$$
\begin{aligned}
\frac{\mathcal{P}_{t}\left(N_{L}, N_{R}, \omega_{L}, \omega_{R}\right)}{\mathcal{P}_{t}\left(-N_{L},-N_{R},-\omega_{L},-\omega_{R}\right)} & =e^{\left(N_{L} \beta_{L} \mu_{L}+N_{R} \beta_{R} \mu_{R}\right)} \\
& \times e^{\left(\beta_{R} \omega_{R}+\beta_{L} \omega_{L}\right)}
\end{aligned}
$$

Here, $\mathcal{P}_{t}\left(N_{L}, N_{R}, \omega_{L}, \omega_{R}\right)=\sum_{n=0,1} \mathcal{P}_{t}\left(n, N_{L}, N_{R}, \omega_{L}, \omega_{R}\right)$. Enforcing energy and charge conservation, $N=N_{L}=-N_{R}$ and $\omega=\omega_{R}=-\omega_{L}$, we recover Eq. (44).

\section{Currents, and measures for vibrational cooling, heating, or instability}

Currents. Analytical expressions for the charge and energy currents are obtained using the definition Eq. (33), utilizing Eqs. (30) and (36). These currents are defined positive when flowing $L$ to $R$, and their closed forms are

$$
\left\langle I_{e}\right\rangle=p_{1}\left(k_{1 \rightarrow 0}^{L \rightarrow R}-k_{1 \rightarrow 0}^{R \rightarrow L}\right)+p_{0}\left(k_{0 \rightarrow 1}^{L \rightarrow R}-k_{0 \rightarrow 1}^{R \rightarrow L}\right),
$$

and

$$
\begin{aligned}
& \left\langle I_{q}\right\rangle= \\
& p_{1}\left[\int_{-\infty}^{\infty} d \omega \omega f_{L}\left(\omega-\omega_{0}\right)\left[1-f_{R}(\omega)\right] J_{L}\left(\omega-\omega_{0}\right) J_{R}(\omega)\right. \\
& \left.-\int_{-\infty}^{\infty} d \omega \omega\left[1-f_{L}\left(\omega+\omega_{0}\right)\right] f_{R}(\omega) J_{L}\left(\omega+\omega_{0}\right) J_{R}(\omega)\right] \\
& +p_{0}\left[\int_{-\infty}^{\infty} d \omega \omega f_{L}\left(\omega+\omega_{0}\right)\left[1-f_{R}(\omega)\right] J_{L}\left(\omega+\omega_{0}\right) J_{R}(\omega)\right. \\
& \left.-\int_{-\infty}^{\infty} d \omega \omega\left[1-f_{L}\left(\omega-\omega_{0}\right)\right] f_{R}(\omega) J_{L}\left(\omega-\omega_{0}\right) J_{R}(\omega)\right] .
\end{aligned}
$$

The TLS population is calculated in the steady-state limit,

$$
p_{1}=\frac{k_{0 \rightarrow 1}^{e}}{k_{0 \rightarrow 1}^{e}+k_{1 \rightarrow 0}^{e}} ; \quad p_{0}=1-p_{1} .
$$

The zero frequency noise current power is given by

$$
\begin{aligned}
& \left\langle S_{e}\right\rangle=-\frac{2}{k_{0 \rightarrow 1}^{e}+k_{1 \rightarrow 0}^{e}}\left\langle I_{e}\right\rangle^{2} \\
& +\frac{4}{k_{0 \rightarrow 1}^{e}+k_{1 \rightarrow 0}^{e}}\left(k_{0 \rightarrow 1}^{L \rightarrow R} k_{1 \rightarrow 0}^{L \rightarrow R}+k_{0 \rightarrow 1}^{R \rightarrow L} k_{1 \rightarrow 0}^{R \rightarrow L}\right) .
\end{aligned}
$$

The energy current, directed towards the vibrational mode, is zero in the steady-state limit, unless the mode is further coupled to a dissipative bath. Formally, it is given by the expression

$$
\left\langle I_{v i b}\right\rangle=-\omega_{0} p_{1}\left[k_{1 \rightarrow 0}^{L \rightarrow R}+k_{1 \rightarrow 0}^{R \rightarrow L}\right]+p_{0} \omega_{0}\left[k_{0 \rightarrow 1}^{L \rightarrow R}+k_{0 \rightarrow 1}^{R \rightarrow L}\right] .
$$

Measures for vibrational instability. The stability of the junction can be estimated, against heating effects, by inspecting several measures. First, following Ref. [16], we define the damping rate $K_{v i b}$ of the vibrational mode as the difference between relaxation and excitation rates,

$$
K_{v i b} \equiv k_{1 \rightarrow 0}^{e}-k_{0 \rightarrow 1}^{e}
$$


Positive $K_{v i b}$ indicates on the "normal" thermal-like behavior, as relaxation processes overcome excitations. In this case, the mode effective temperature (defined below) is found to be either below (cooling) or above (heating) the environmental temperature, yet the junction remains stable in the sense that the ground vibrational state population is larger than the excited level population. A negative value for $K_{v i b}$ evinces on the process of an uncontrolled heating of the molecular mode, eventually leading to junction instability and breakdown. One can also directly inspect the TLS population: population inversion reflects on vibrational instability.

Effective temperature. The TLS population can be further utilized as a measure for the molecular vibration effective temperature, $1 / \beta_{\text {eff }}$, defined using an equilibrium relation,

$$
\frac{p_{1}}{p_{0}}=e^{-\beta_{e f f} \omega_{0}} .
$$

A negative value for $\beta_{\text {eff }}$ attests on population inversion, thus junction instability. When $\beta_{\text {eff }}$ is positive, one should compare it to the reservoirs' inverse temperature $\beta$ : If $\beta_{\text {eff } f}>\beta$ the system demonstrates bias-induced cooling phenomena. For $\beta_{\text {eff }}<\beta$ the vibrational mode is heated up relative to its environment. The latter typically occurs at an intermediate bias voltage, before instabilities take place.
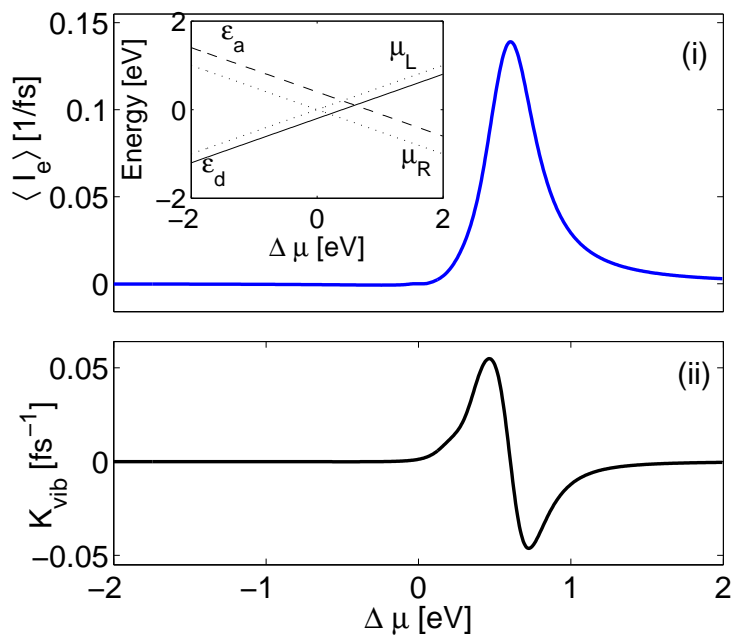

FIG. 3: (i) Charge current in a rectifying molecular junction. Inset: Energies of the donor (full line) and acceptor states (dashed line). The dotted lines correspond to the chemical potential at the left and right sides. (ii) Damping rate $K_{v i b}$. The junction parameters are $\Gamma_{\nu}=0.2,1 / \beta_{\nu}=0.005, \kappa=0.1$, $\omega_{0}=0.05$ and $\epsilon_{d}(\Delta \mu=0)=-0.2, \epsilon_{a}(\Delta \mu=0)=0.4$, all in units of $[\mathrm{eV}]$.

\section{E. Numerical results: isolated mode}

We demonstrate cooling, heating and mode instability upon varying the bias voltage. A generic mechanism

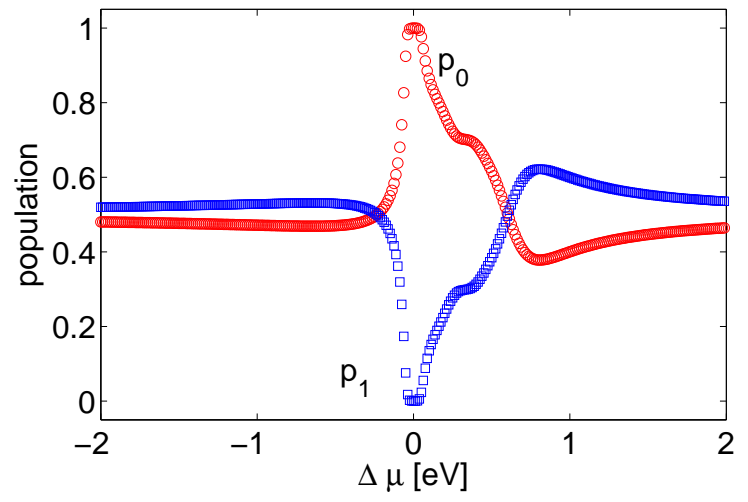

FIG. 4: Population of the two-state "vibration" as a function of bias voltage. Parameters are the same as in Fig. 3.

leading to vibrational instabilities (and eventually junction rupture) in D-A molecular rectifiers has been discussed in Ref. [16]: At large positive bias, when the D state is positioned above the acceptor level, electron-hole pair excitations by the molecular vibration (TLS here) dominate the mode dynamics. This can be schematically seen in Fig. 2, where the rate $k_{0 \rightarrow 1}^{L \rightarrow R}$ overcomes other rates once the donor spectral function is positioned above the acceptor spectral function. As $K_{v i b}$ becomes negative, population inversion is observed.

The junction setup is displayed in Fig. 3. D and A levels are positioned such that in equilibrium, $\Delta \mu=0$, the donor level is placed below the Fermi energy $\mu$, while the acceptor level is of a higher energy, $\epsilon_{d}(\Delta \mu=0)<$ $\mu<\epsilon_{a}(\Delta \mu=0)$. Under an applied bias, the levels are assumed to linearly follow the external potential drive (inset) [45]. Therefore, at a particular positive bias the levels cross. Beyond that, the levels exchange arrangement, and the $\mathrm{D}$ state is of a higher energy. Throughout the paper, the parameters $\omega_{0}, \Gamma_{\nu}, \Gamma_{p h}, 1 / \beta, \kappa, \epsilon_{d, a}$ and $\Delta \mu$ are given in units of $\mathrm{eV}$.

The junction's current-voltage characteristics is displayed in Fig. 3 (i), manifesting a substantial rectification effect. For negative polarity, $\Delta \mu=\mu_{L}-\mu_{R}<0$, the current is rather small. In contrast, for positive bias the current substantially increases once $\Delta \mu>\omega_{0}$, reaching a maximum when the energy levels satisfy $\epsilon_{d}-\epsilon_{a} \sim \omega_{0}$. Level broadening, $\Gamma_{\nu}$, affects the actual position of the maximum. The damping rate, $K_{v i b}$, is displayed in Fig. 3(ii). It shows the following features: First, for large negative bias, $\Delta \mu<-0.2, K_{v i b}$ is negative. This instability can be immediately removed, once a very weak coupling to a phononic thermal reservoir is turned on, see Figs. 6 and 11 below. Beyond that, the damping rate $K_{v i b}$ is positive between $-0.2 \lesssim \Delta \mu \lesssim 0.6$, indicating on a stable mode of operation. However, for large enough bias, $\Delta \mu \gtrsim 0.6$, once $\epsilon_{d}>\epsilon_{a}$, uncontrolled TLS heating takes place, recognized by a sign change in $K_{v i b}$. It should be noted that the instability takes place in the parameter range very relevant to the rectifier operation. It is thus important to understand how to tune the system config- 
uration so as to sustain junction functionality.

Fig. 4 depicts the corresponding population of the two levels. At zero bias, $k_{0 \rightarrow 1}^{e}=0$, thus the population of the excited state is identically zero. At low positive bias one finds that $k_{0 \rightarrow 1}^{e}<k_{1 \rightarrow 0}^{e}$, leading to the "normal" situation of $p_{0}>p_{1}$. However, once the bias is large and the donor state is positioned above the acceptor site, $(\Delta \mu \sim 0.6)$ the excitation rate $k_{0 \rightarrow 1}^{e}$ exceeds the relaxation rate $k_{1 \rightarrow 0}^{e}$ and population inversion takes place. We note that for a negative bias, small population inversion is also observed, as electrons damp energy to the TLS when crossing the junction. However, since $\left\langle I_{e}\right\rangle$ is rather small (Fig. 3), we do not expect molecular instability in this regime, see also Fig. 11.

The details of the damping rate $K_{v i b}$ depend on the level broadening and the reservoirs temperature as we show in Fig. 5. The position of the turnover, between positive to negative damping, appears at a similar value for the bias, and it is generally independent of the reservoirs temperatures and $\Gamma_{\nu}$. However, the width of the curve largely depends on these parameters.

It should be noted that the development of the instability, as reported in Figs. 3, 4 and 5, does not depend on the concrete value of $\kappa$, the strength of the moleculemode coupling, and the behavior persists in the limit of vanishing vibronic coupling, $\kappa \rightarrow 0$. In the next section we allow the vibrational mode to thermalize with a phononic environment at a rate $\Gamma_{p h}$. In this case, the competition between $\kappa$ and $\Gamma_{p h}$ determines the onset of instability, see Eq. (58).

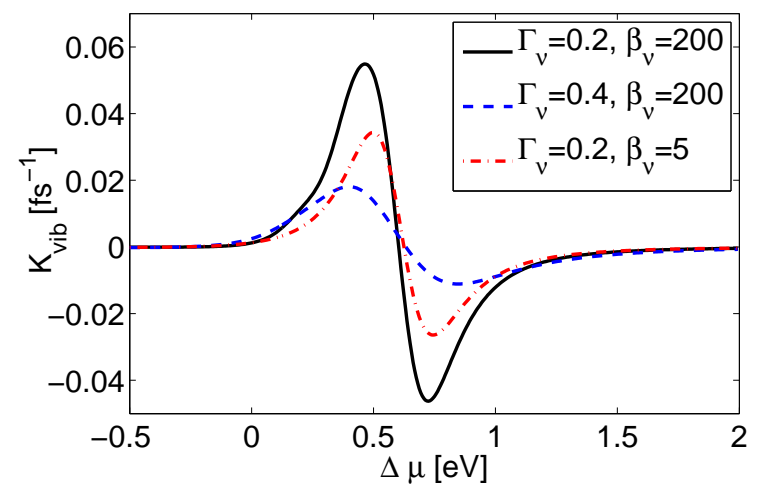

FIG. 5: Damping rate in a rectifying junction for different broadening parameters, $\Gamma_{\nu}=0.2, \beta_{\nu}=200$ (full) $\Gamma_{\nu}=0.4$, $\beta_{\nu}=200$ (dashed) $\Gamma_{\nu}=0.2, \beta_{\nu}=5$ (dashed-dotted). Other parameters are the same as in Fig. 3 .

\section{F. Numerical results: dissipative mode}

Up to this point, we have assumed that the molecular vibrational mode (TLS here) is well isolated from other vibrations. In reality, internal modes typically exchange energy with "secondary" reservoirs modes, either internal, or part of a larger environment, opening up an additional route for energy dissipation. It is expected that in the presence of such a thermal bath, the region of vibrational instability $\left(K_{v i b}<0\right)$ would become limited.

A simple model that is capable of describing a hierarchy of energy transfer processes, electronic energy $\rightarrow$ specific vibrational excitation $\rightarrow$ thermal bath, is given by an extension of the model (13),

$$
\begin{aligned}
H_{A+B} & =\frac{\omega_{0}}{2} \sigma_{z}+\sigma_{x}\left(F_{e}+F_{b}\right) \\
& +\sum_{l} \epsilon_{l} a_{l}^{\dagger} a_{l}+\sum_{r} \epsilon_{r} a_{r}^{\dagger} a_{r}+\sum_{\alpha} \omega_{\alpha} b_{\alpha}^{\dagger} b_{\alpha} .
\end{aligned}
$$

The notation " $H_{A+B}$ " indicates that the anharmonic mode is coupled to a thermal bath (B). The operator $F_{e}$ describes electron-hole pair excitations as in Eq. (14). The thermal bath operator, coupled to the TLS transitions, includes displacements of reservoir modes,

$$
F_{b}=\sum_{\alpha} v_{\alpha}\left(b_{\alpha}^{\dagger}+b_{\alpha}\right)
$$

with $b_{\alpha}^{\dagger}\left(b_{\alpha}\right)$ as a bosonic creation (annihilation) operator for the $\alpha$ phonon-reservoir mode.

Derivation of the full counting statistics can be reiterated, while including energy dissipation from the TLS to the phonon bath. For details, see Appendix A. We find that the expression for the charge current stays intact, satisfying the formal expression (48). However, the steady-state populations are corrected by a phonon relaxation rate constant as

$$
p_{1}=\frac{k_{0 \rightarrow 1}^{e}+\Gamma_{p h}\left(\omega_{0}\right) n_{p h}\left(\omega_{0}\right)}{k_{0 \rightarrow 1}^{e}+k_{1 \rightarrow 0}^{e}+\Gamma_{p h}\left(\omega_{0}\right)\left[2 n_{p h}\left(\omega_{0}\right)+1\right]} .
$$

The electronic transition induced rates $k_{n \rightarrow n^{\prime}}^{e}$ are those defined in Eq. (19); the phononic relaxation rate constant is $\Gamma_{p h}(\omega)=2 \pi \sum_{\alpha} v_{\alpha}^{2} \delta\left(\omega_{\alpha}-\omega\right)$. The function $n_{p h}(\omega)=\left[e^{\beta_{p h} \omega}-1\right]^{-1}$ stands for the Bose-Einstein distribution with $\beta_{p h}$ as the temperature of the phonon bath.

Fig. 6 presents the steady-state population for two choices of $\Gamma_{p h}$. When this parameter is small, population inversion still takes place around donor-acceptor level crossing. However, the phenomenon disappears at large enough bias. Thus, quite interestingly, the domain of instability extends intermediate bias values, while the system becomes stable again at very high bias. This can be reasoned by inspecting $K_{v i b}$. It is defined as the difference between TLS relaxation and excitation rate constants. In the presence of a thermal bath it is given by

$$
\begin{aligned}
K_{v i b} & =\left(k_{1 \rightarrow 0}^{e}+\Gamma_{p h}\left(n_{p h}+1\right)\right)-\left(k_{0 \rightarrow 1}^{e}+\Gamma_{p h} n_{p h}\right) . \\
& =k_{1 \rightarrow 0}^{e}-k_{0 \rightarrow 1}^{e}+\Gamma_{p h} .
\end{aligned}
$$

For convenience, the $\omega_{0}$ dependence of the rates is left out. While $k_{1 \rightarrow 0}^{e}<k_{0 \rightarrow 1}^{e}$ may hold at large bias, both these rates diminish with $\Delta \mu$, and the net damping rate can become positive due to the $\Gamma_{p h}$ contribution. For large enough $\Gamma_{p h}$, instability does not take place at any voltage. 


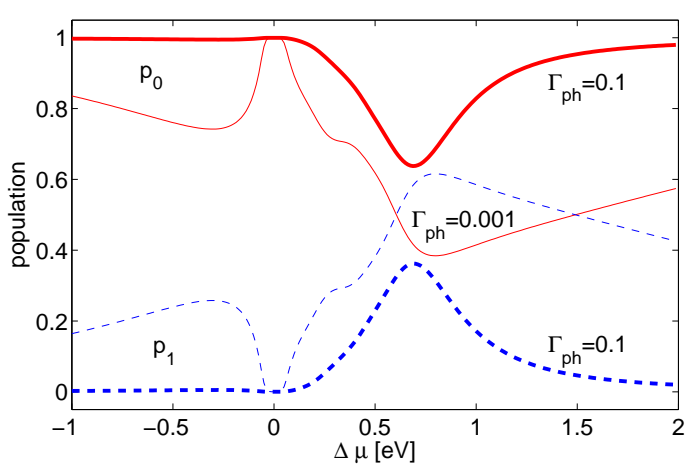

FIG. 6: TLS population as a function of bias voltage for $\Gamma_{p h}=$ 0.001 (narrow lines) and $\Gamma_{p h}=0.1$ (heavy lines). The excited (ground) state population is presented by dashed (full) lines. Other parameters are the same as in Fig. 3 with $\beta_{p h}=200$.

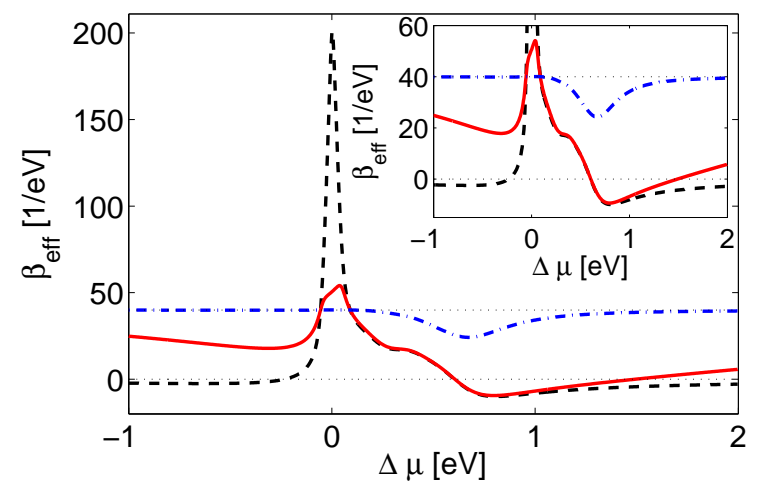

FIG. 7: Effective TLS temperature, $\Gamma_{p h}=0$ (dashed line); $\Gamma_{p h}=0.001$ and $\beta_{p h}=40$ (full line) and $\Gamma_{p h}=0.4$ and $\beta_{p h}=40$ (dashed-dotted line). The inset zooms on the latter two cases. The dotted lines mark the values $\beta_{p h}=40$ and $\beta=0$. Other junction parameters are the same as in Fig. 3, with $\beta_{\nu}=200$.

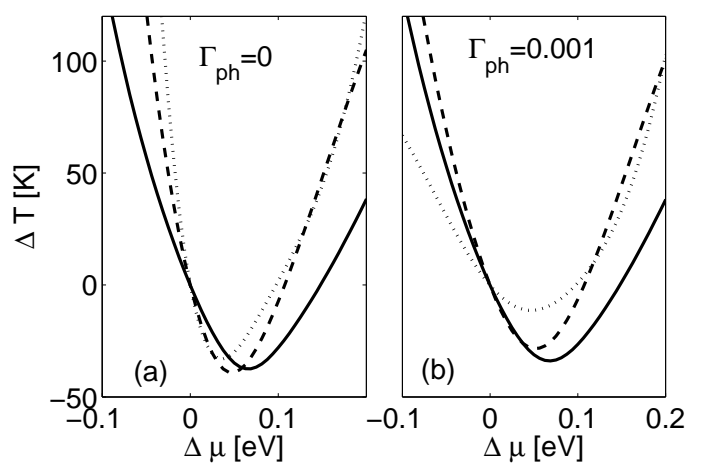

FIG. 8: Cooling of the molecular vibration for $\omega_{0}=0.05$ (dotted line), $\omega_{0}=0.15$ (dashed line), $\omega_{0}=0.3$ (full line). (a): $\Gamma_{p h}=0$. (b): $\Gamma_{p h}=0.001$. Other junction parameters are $\Gamma_{L}=\Gamma_{R}=0.1$, and $\beta_{p h}=\beta_{\nu}=40$. The levels are shifted with the bias voltage as depicted in Fig. 3.

The effective TLS temperature, defined in Eq. (54), is displayed in Fig. 7 for several cases. First, in the absence of a phonon thermal bath we find that at zero bias voltage the molecular mode is thermalized at the metals' temperature, $\beta_{\text {eff }}=\beta$. This effective inverse temperature quickly drops with increasing bias, becoming negative around the value of $\Delta \mu=0.6$, where the D-A levels cross. This behavior indicates on the instability of the junction from that point. Next, we weakly couple $\left(\Gamma_{p h}=0.001\right)$ the single mode to an additional thermal bath maintained at $\beta_{p h}=40$. The following observations can be made: (i) For negative bias, the mode is close to be equilibrated with the phonon bath, as electron-hole excitations are sparse. (ii) In accordance with Fig. $6, \beta_{\text {eff }}$ can reach a negative (unstable) value around $\Delta \mu=0.6$. However, $\beta_{\text {eff }}$ becomes positive at large enough bias, indicating that the system re-enter a stability region. (iii) At low bias, $-0.05<\Delta \mu<0.1$, the mode temperature is lower than its phononic environment, as $\beta_{\text {eff }}>\beta_{p h}$. (iv) At strong mode-thermal bath coupling, $\Gamma_{p h}=0.4$, the mode is closed to be thermalized with $\beta_{p h}$ at all biases.

We now demonstrate mode cooling, to a temperature below the phonon bath and metals temperature. Keeping both electron and phonon reservoirs at a fixed temperature of $\beta=40$, the temperature difference $\Delta T \equiv$ $T_{\text {eff }}-T_{p h}$ is presented in Fig. 8, for various frequencies and $\Gamma_{p h}$ values. Generally, we note that at low positive bias, $\Delta \mu<0.1$, one may cool the mode by $40 \mathrm{~K}$, the result of its coupling to a nonequilibrium environment.

\section{HARMONIC-MODE RECTIFIER}

We study next the dynamics of model (11), assuming a harmonic mode coupled to the electronic system. The relevant equations of motion for the mode levels population are [38]

$$
\begin{aligned}
\dot{p}_{n} & =-\left[n k_{d}^{e}+(n+1) k_{u}^{e}\right] p_{n} \\
& +(n+1) k_{d}^{e} p_{n+1}+n k_{u}^{e} p_{n-1}
\end{aligned}
$$

Here, the decay rate constant is independent of the level index $k_{d}^{e} \equiv k_{1 \rightarrow 0}^{e}$, and similarly, $k_{u}^{e} \equiv k_{0 \rightarrow 1}^{e}$, defined in Eq. (19). In order to calculate the CGF, we define $\mathcal{P}_{t}(n, N, \omega)$ as the probability that by the time $t$ the harmonic mode occupies level $n, N$ electrons have been added to the $R$ bath and an additional energy $\omega$ has been acquired by the $R$ bath. This quantity follows a differential equation analogous to Eq. (27). The characteristic function is an array whose $n$th element is $|\mathcal{Z}(\chi, \eta, t)\rangle_{n}=\sum_{N} e^{i N \chi} \int_{-\infty}^{\infty} \mathcal{P}_{t}(n, N, \omega) e^{i \omega \eta} d \omega$. It satisfies a differential equation corresponding to Eq. (29)

$$
\frac{d|\mathcal{Z}(\chi, \eta, t)\rangle}{d t}=-\hat{\mathcal{W}}(\chi, \eta)|\mathcal{Z}(\chi, \eta, t)\rangle
$$

with the $n \times n$ matrix $\hat{\mathcal{W}}(\chi, \eta)$, 


$$
\begin{aligned}
& \hat{\mathcal{W}}(\chi, \eta)=
\end{aligned}
$$

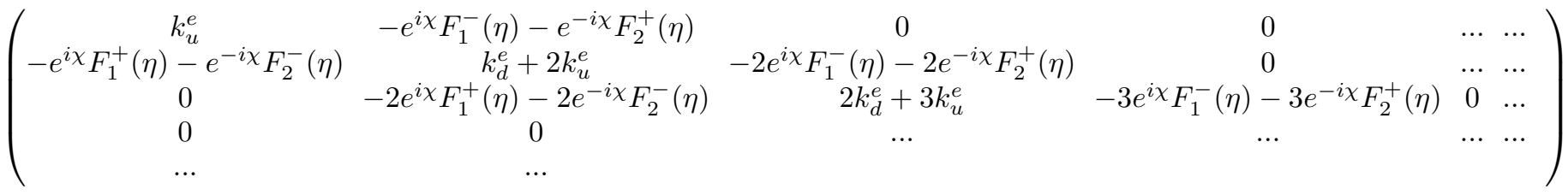

We can readily confirm the fluctuation theorem, by inspecting the eigenvalues of $\operatorname{det}[\lambda I-\hat{\mathcal{W}}]$. For convenience, we define the auxiliary matrix $A \equiv \lambda I-\hat{\mathcal{W}}$. Since it is tridiagonal, its determinant can be evaluated in a recursive manner as

$$
\begin{aligned}
\operatorname{det}[A]_{1, \ldots, n} & =a_{n, n} \operatorname{det}[A]_{1, \ldots, n-1} \\
& -a_{n, n-1} a_{n-1, n} \operatorname{det}[A]_{1, \ldots, n-2}
\end{aligned}
$$

where $[A]_{1, \ldots, k}$ denotes the submatrix constructed by the first $k$ rows and columns of $A$. Thus, the symmetry of $\operatorname{det}[A]$ with respect to $\chi$ and $\eta$ is determined by the symmetry of the products $a_{n, n-1} a_{n-1, n}=w_{n, n-1} w_{n-1, n}$, with $w_{i, j}$ the matrix elements of $\hat{\mathcal{W}}$,

$$
\begin{aligned}
& d_{n}(\chi, \eta) \equiv w_{n, n-1}(\chi, \eta) w_{n-1, n}(\chi, \eta) \propto \\
& {\left[e^{i \chi} F_{1}^{-}(\eta)+e^{-i \chi} F_{2}^{+}(\eta)\right]\left[e^{i \chi} F_{1}^{+}(\eta)+e^{-i \chi} F_{2}^{-}(\eta)\right]}
\end{aligned}
$$

Using the relations (39)-(40), we conclude that

$$
d_{n}(\chi, \eta)=d_{n}\left(-\chi+i\left(\beta_{L} \mu_{L}-\beta_{R} \mu_{R}\right),-\eta+i \Delta \beta\right) .
$$

Given the recursive nature of $\operatorname{det}[A]$, this symmetry holds for all the eigenvalues of $\hat{\mathcal{W}}$, confirming the fluctuation theorem (37). We now obtain the steady-state population of the harmonic mode, by solving Eq. (59) in the long time limit, $\dot{p}_{n}=0$. This results in [38]

$$
p_{n}=\left(\frac{k_{u}^{e}}{k_{d}^{e}}\right)^{n} \frac{1}{\sum_{n=0}^{\infty}\left(k_{u}^{e} / k_{d}^{e}\right)^{n}} ; \quad n=0,1 \ldots, \infty
$$

or

$$
p_{n}=\left(\frac{k_{u}^{e}}{k_{d}^{e}}\right)^{n}\left(1-\frac{k_{u}^{e}}{k_{d}^{e}}\right)
$$

if $k_{u}^{e}<k_{d}^{e}$. In the opposite limit, the system passes into the unstable regime, and the levels' population diverges. In that sense, the harmonic model is unphysical as the number of states is not bounded. One way to pull the system back into physical realm is to couple the vibrational mode with a thermal bath, see Appendix A. As explained above for the TLS-mode case, the following damping rate is a measure for the junction stability,

$$
K_{v i b}=k_{d}^{e}-k_{u}^{e}
$$

A negative value indicates on junction instability, as uncontrolled heating of the mode takes place. Using steadystate populations, we proceed and derive the charge current expression, valid only if $k_{u}^{e}<k_{d}^{e}$,

$$
\begin{aligned}
\left\langle I_{e}\right\rangle & =\frac{1}{t}\left\langle 11 \ldots\left|\frac{\partial}{\partial i \chi} e^{-\hat{\mathcal{W}}(\chi, \eta) t}\right|_{\chi=0, \eta=0} \mid \mathcal{Z}(\chi, \eta, t=0)\right\rangle \\
& =-\left\langle 11 \ldots\left|\frac{\partial \hat{\mathcal{W}}}{\partial i \chi}\right|_{\chi=0, \eta=0} \mid P_{s s}\right\rangle \\
& =-\left[\frac{\left(k_{d}^{R \rightarrow L}-k_{d}^{L \rightarrow R}\right) k_{u}^{e}}{k_{d}^{e}-k_{u}^{e}}+\frac{\left(k_{u}^{R \rightarrow L}-k_{u}^{L \rightarrow R}\right) k_{d}^{e}}{k_{d}^{e}-k_{u}^{e}}\right] \\
& =-2 \frac{k_{u}^{R \rightarrow L} k_{d}^{R \rightarrow L}-k_{u}^{L \rightarrow R} k_{d}^{L \rightarrow R}}{k_{d}^{e}-k_{u}^{e}}
\end{aligned}
$$

Here, $P_{s s}$ is a vector of steady-state population given by Eq. (66). Equations (59)-(68) can be generalized to include the interaction of the harmonic mode with a dissipative-thermal phonon bath. Appendix A exemplifies this procedure for the anharmonic-mode model. In practice, the electronic induced rates $k_{d}^{e}$ and $k_{u}^{e}$ in Eq. (66) are augmented by a phononic contribution, $\Gamma_{p h}\left(\omega_{0}\right)\left[n_{p h}\left(\omega_{0}\right)+1\right]$ and $\Gamma_{p h}\left(\omega_{0}\right) n_{p h}\left(\omega_{0}\right)$, respectively.

Fig. 9 displays the charge current for zero and finite $\Gamma_{p h}$ strength. In the absence of coupling to the phonon bath, the current diverges around $\Delta \mu=0.6$, where instability occurs. For finite $\Gamma_{p h}$, the current is larger when the vibrational mode is harmonic, compared to the TLSmode case (dotted), as the electronic energy can be used to excite multiple transitions.

Fig. 10 further demonstrates the "stabilizing" effect the interaction with a heat bath has on the harmonic mode. We display the population for the states $n=$ 0 to $n=3$, top to bottom. For $\Gamma_{p h}=0$ the data is presented up to $\Delta \mu=0.6$, where the population becomes unphysical (levels' population goes to zero there since infinite number of vibrational states are occupied). This point is indicated by an arrow.

The cooling and heating behavior depicted in Fig. 8 for the TLS model could be repeated for the present harmonic-mode case as well, to yield the same behavior. The reason is that $\beta_{\text {eff }}$ is determined by the ratio of rates, and this ratio is identical in the two models. Our conclusion here is thus that including modeanharmonicity is important for transport calculations: Harmonic-mode model can lead to unphysical results 


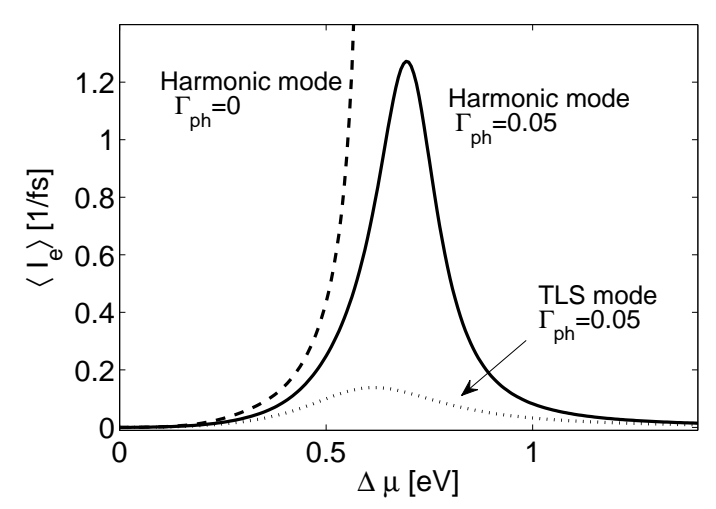

FIG. 9: Charge current in the harmonic-mode model for $\Gamma_{p h}=0$ (dashed line) and $\Gamma_{p h}=0.05$ (full line). For comparison, we also present the current in the TLS-mode model with $\Gamma_{p h}=0.05$ (dotted line). $\beta_{\nu}=\beta_{p h}=40$, other parameters are the same as in Fig. 3.

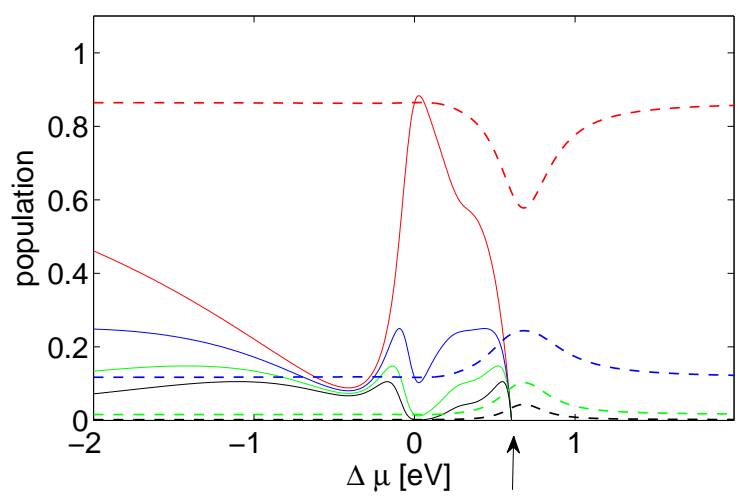

FIG. 10: Population of the first four levels of the harmonic mode. Full lines: weak interaction with the heat bath, $\Gamma_{p h}=$ $10^{-4}$. The population becomes unphysical (negative) for $\Delta \mu>0.6$. Dashed lines: strong interaction with heat bath, $\Gamma_{p h}=0.1$, lifts the instability. $\beta_{\nu}=\beta_{p h}=40$, other parameters are the same as in Fig. 3.

(e.g., current divergence) since there is no saturation situation for the vibrational mode. In particular, including molecular anharmonic aspects is essential for obtaining reliable results when simulating junction behavior close to the critical bias, where an instability occurs.

\section{SUMMARY}

We have studied vibrational cooling, heating, and instability formation in a phonon assisted D-A electron rectifier junction using a full-counting statistics approach. Variants of the basic model were constructed, assuming either harmonic or an anharmonic vibrational mode, further allowing energy dissipation to a phononic thermal environment.

Putting together our observations, we present in figures 11 and 12 stability maps for the system; the dark region codes instability zones, with negative $K_{v i b}$. These diagrams hold for both TLS and harmonic mode cases. A reentrant behavior is observed in Fig. 11: For a fixed value of $\Gamma_{p h}$, say $\Gamma_{p h}=0.005$, the junction is stable for $\Delta \mu<0.6$, unstable around $0.6<\Delta \mu<1.2$, while beyond that, the junction is operative again. The reason for this behavior is that electronic-induced excitation and relaxation rates, $k_{u}^{e}$ and $k_{d}^{e}$, become both small at large bias, thus the thermal bath-induced rates dominate the mode dynamics, leading to a normal-thermal like behavior.

The coupling of the D and A molecular states to the metal leads may be further tuned experimentally. In Fig. 12 we show a stability map of the junction as a function of voltage bias and metal-molecule hybridization $\Gamma_{L}=\Gamma_{R}$. We explore two situations: (a) The molecular mode is perfectly isolated from other vibrations, and (b) $\Gamma_{p h}$ is finite. In both cases, once metal-molecule coupling is large enough, a stable operation sustains. This result seems initially counterintuitive, as one expects strongly coupled molecules to support high charge and energy currents, potentially leading to junction rupture. However, the key factor in the formation of vibrational instability here is the fact that at certain voltages the vibrational excitation rate $k_{u}^{e}$ exceeds the relaxation rate $k_{d}^{e}$. Inspecting the rates (25), one can analytically prove that if the effective density of states is energy independent, $J_{\nu}(\epsilon)=C$, which is the case at strong metal-molecule coupling, then $k_{d}^{e}-k_{u}^{e} \propto C^{2} \omega_{0}$, a positive number. The key factor in instability build-up is thus the usage of electronic reservoirs with effective DOS [Eq. (24)] peaked around different energies, the D and A levels.

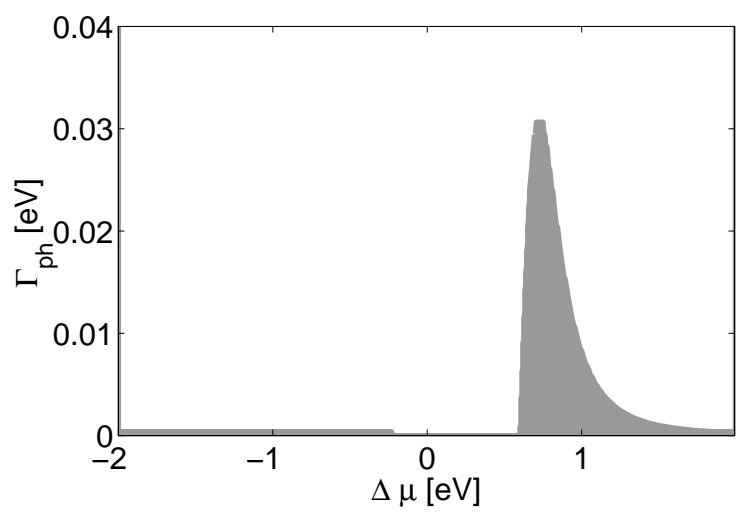

FIG. 11: Stability diagram. The dark island and the narrow strip (at negative bias) are the parametric region in which the junction becomes unstable. Other parameters are the same as in Fig. 3, besides the temperatures, $\beta_{L}=\beta_{R}=\beta_{p h}=40$.

Concluding our observations: (i) We confirmed the steady-state entropy production FT for the different model variants. This is a non-trivial task since charge and energy currents here are not tightly coupled, a result of the strong metal-D and A-metal couplings. Therefore, one needs to separately count particle number and energy transfer in the system. (ii) We derived simple 

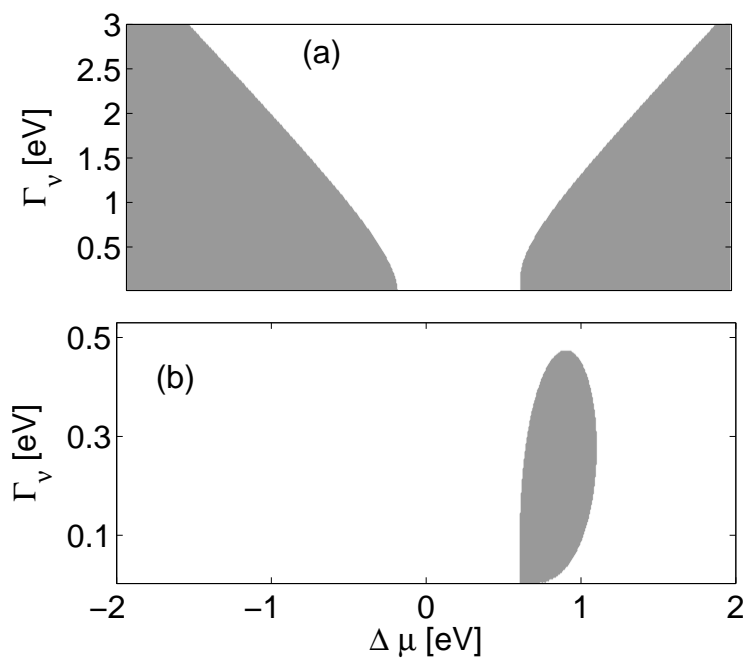

FIG. 12: (a): Stability diagram for $\Gamma_{p h}=0$. (b): Stability diagram with $\Gamma_{p h}=0.005$. The dark region is the parametric region in which the junction becomes unstable, $K_{v i b}<0$. Other parameters are the same as in Fig. 3, besides the temperatures $\beta_{L}=\beta_{R}=\beta_{p h}=40$.

analytical expressions for the charge current, assuming either harmonic or an anharmonic vibrational mode. As expected, harmonic-mode junctions better conduct since the electronic energy can be used to excite multiple vibrational states. An anharmonic mode quickly reaches saturation. (iii) We defined an effective temperature for the vibration and demonstrated bias induced cooling at low bias, $\Delta \mu<0.2$. (iv) At intermediate voltage bias, $0.2<\Delta \mu<0.6$, heating effects were observed, and the mode effective temperature exceeds the environmental temperature. (v) Once the donor and acceptor levels switch position for $\Delta \mu>0.6, \epsilon_{d}>\epsilon_{a}$, instability develops: The mode excitation rate exceeds the relaxation rate, and the vibrational mode uncontrollably heats. (vi) Coupling the vibrational mode to an external thermal bath stabilizes the junction. In particular, assuming a weak interaction to a phonon bath, $\Gamma_{p h}=0.005$, junction instability is removed for $\Delta \mu>1.2$; the electronicinduced rates diminish and the mode dynamics is controlled by the thermal bath. (vii) The appearance of vibrational instability can be traced down to the metals' energy dependent DOS, different at the two ends.

The simple models described here elucidate the role of different factors on vibrational cooling, heating, and instability build-up in a D-A electronic rectifier. The effects of mode frequency, its interaction with other modes, the reservoirs' temperature, metal-molecule coupling strength, and bias voltage, were examined. While the focus of this work has been on vibrational effects, the theory developed here could be used for describing the coupling of an electronic junction to a cavity modeelectromagnetic environment. One could thus reformulate this study and describe cooling, heating and diodelike effects in photonic heat conduction [48-52]. Future work will be devoted to the study of noise processes in phonon-assisted tunneling junctions, with the motivation to expose mode properties (harmonicity) through the noise characteristics.

\section{Acknowledgments}

DS acknowledges support from an NSERC discovery grant. The work of LS was supported by an Early Research Award of DS.

\section{Appendix A: Full counting statistics for charge and energy in the dissipative anharmonic-mode rectifier model}

We describe here the derivation of the generating function and the charge current for the anharmonic-mode bath-coupled rectifier model,

$$
\begin{aligned}
H_{A+B} & =\frac{\omega_{0}}{2} \sigma_{z}+\sigma_{x}\left(F_{e}+F_{b}\right) \\
& +\sum_{l} \epsilon_{l} a_{l}^{\dagger} a_{l}+\sum_{r} \epsilon_{r} a_{r}^{\dagger} a_{r}+\sum_{\alpha} \omega_{\alpha} b_{\alpha}^{\dagger} b_{\alpha} \cdot(
\end{aligned}
$$

Here, the TLS excitation and relaxation processes are coupled to both electronic transitions in the junction and to a thermal-phononic reservoir,

$$
\begin{aligned}
& F_{e}=\kappa \sum_{l, r}\left(\lambda_{l}^{*} \lambda_{r} a_{l}^{\dagger} a_{r}+\lambda_{r}^{*} \lambda_{l} a_{r}^{\dagger} a_{l}\right) \\
& F_{b}=\sum_{\alpha} v_{\alpha}\left(b_{\alpha}^{\dagger}+b_{\alpha}\right)
\end{aligned}
$$

$a_{l}, a_{r}$ are fermionic annihilation operators corresponding to the left and right metals; the coefficients $\lambda_{\nu}$ were defined in Eq. (8), $\kappa$ denotes the D-A tunneling strength, $b_{\alpha}$ is a bosonic operator, describing the $\alpha$ reservoir mode, $v_{\alpha}$ quantifies the TLS-bath interaction strength. For more details, see text around Eq. (55).

The impurity (TLS) dynamics can be obtained by using a master-equation approach [43]. The procedure involves a second order perturbation theory in the impurity coupling to both electronic and phononic reservoirs. In the markovian limit, under the rotating wave approximation, one standardly achieves kinetic equations that separately account for the electronic (e) and phononic (b) relaxation pathways,

$$
\begin{aligned}
& \dot{p}_{1}=-\left(k_{1 \rightarrow 0}^{e}+k_{1 \rightarrow 0}^{b}\right) p_{1}+\left(k_{0 \rightarrow 1}^{e}+k_{0 \rightarrow 1}^{b}\right) p_{0} \\
& p_{1}+p_{0}=1 .
\end{aligned}
$$

The relaxation terms are given by

$$
\begin{aligned}
& k_{n \rightarrow n^{\prime}}^{e}=\int_{-\infty}^{\infty} e^{i\left(\epsilon_{n}-\epsilon_{n^{\prime}}\right) \tau}\left\langle F_{e}(\tau) F_{e}(0)\right\rangle d \tau \\
& k_{n \rightarrow n^{\prime}}^{b}=\int_{-\infty}^{\infty} e^{i\left(\epsilon_{n}-\epsilon_{n^{\prime}}\right) \tau}\left\langle F_{b}(\tau) F_{b}(0)\right\rangle d \tau .
\end{aligned}
$$


Here, $\epsilon_{n}$ is the energy of the $n$th vibrational level. Electron induced rate constants are detailed through Eqs. (19)-(25). The thermal bath induced rates can be similarly put together,

$$
\begin{aligned}
k_{1 \rightarrow 0}^{b} & =\Gamma_{p h}\left(\omega_{0}\right)\left[n_{p h}\left(\omega_{0}\right)+1\right], \\
k_{0 \rightarrow 1}^{b} & =k_{1 \rightarrow 0}^{b} e^{-\omega_{0} \beta_{p h}}
\end{aligned}
$$

$n_{p h}(\omega)=\left[e^{\beta_{p h} \omega}-1\right]^{-1}$ denotes the Bose-Einstein distribution function and $\Gamma_{p h}(\omega)=2 \pi \sum_{\alpha} v_{\alpha}^{2} \delta\left(\omega_{\alpha}-\omega\right)$. For brevity, we ignore below the direct reference to frequency. We now define the probability distribution func- tion $\mathcal{P}_{t}\left(n, N, \omega_{L}, \omega_{R}, q \omega_{0}\right)$, as the probability to find the system at time $t$ in state $n=0,1$, with $N$ electrons transferred to the right bath, $\omega_{\nu}$ excess energy accumulated at the $\nu$ bath $(\nu=L, R)$, and $q \omega_{0}$ energy attained by the phonon bath, due to the transfer of $q$ quantas from the TLS to this bath. Note that charge conservation between the $L$ and $R$ baths is enforced, allowing us to work with a single counting field for describing charge transfer processes in the steady-state limit.

We now resolve the associated master equation for the two-state population, to its charge and energy contributions. The resulting equations are analogous to Eq. (27),

$$
\begin{aligned}
\dot{\mathcal{P}}_{t}\left(1, N, \omega_{L}, \omega_{R}, q \omega_{0}\right) & =-\mathcal{P}_{t}\left(1, N, \omega_{L}, \omega_{R}, q \omega_{0}\right)\left[k_{1 \rightarrow 0}^{e}+\Gamma_{p h}\left(\omega_{0}\right)\left[n_{p h}\left(\omega_{0}\right)+1\right]\right] \\
& +\int_{-\infty}^{\infty} \mathcal{P}_{t}\left(0, N-1, \omega_{L}+\epsilon, \omega_{R}-\epsilon+\omega_{0}, q \omega_{0}\right) f_{L}(\epsilon)\left[1-f_{R}\left(\epsilon-\omega_{0}\right)\right] J_{L}(\epsilon) J_{R}\left(\epsilon-\omega_{0}\right) d \epsilon \\
& +\int_{-\infty}^{\infty} \mathcal{P}_{t}\left(0, N+1, \omega_{L}-\epsilon+\omega_{0}, \omega_{R}+\epsilon, q \omega_{0}\right) f_{R}(\epsilon)\left[1-f_{L}\left(\epsilon-\omega_{0}\right)\right] J_{R}(\epsilon) J_{L}\left(\epsilon-\omega_{0}\right) d \epsilon \\
& +\mathcal{P}_{t}\left(0, N, \omega_{L}, \omega_{R},(q+1) \omega_{0}\right) \Gamma_{p h}\left(\omega_{0}\right) n_{p h}\left(\omega_{0}\right) \\
\dot{\mathcal{P}}_{t}\left(0, N, \omega_{L}, \omega_{R}, q \omega_{0}\right) & =-\mathcal{P}_{t}\left(0, N, \omega_{L}, \omega_{R}, q \omega_{0}\right)\left[k_{0 \rightarrow 1}^{e}+\Gamma_{p h}\left(\omega_{0}\right) n_{p h}\left(\omega_{0}\right)\right] \\
& +\int_{-\infty}^{\infty} \mathcal{P}_{t}\left(1, N-1, \omega_{L}+\epsilon, \omega_{R}-\epsilon-\omega_{0}, q \omega_{0}\right) f_{L}(\epsilon)\left[1-f_{R}\left(\epsilon+\omega_{0}\right)\right] J_{L}(\epsilon) J_{R}\left(\epsilon+\omega_{0}\right) d \epsilon \\
& +\int_{-\infty}^{\infty} \mathcal{P}_{t}\left(1, N+1, \omega_{L}-\epsilon-\omega_{0}, \omega_{R}+\epsilon, q \omega_{0}\right) f_{R}(\epsilon)\left[1-f_{L}\left(\epsilon+\omega_{0}\right)\right] J_{R}(\epsilon) J_{L}\left(\epsilon+\omega_{0}\right) d \epsilon \\
& +\mathcal{P}_{t}\left(1, N, \omega_{L}, \omega_{R},(q-1) \omega_{0}\right) \Gamma_{p h}\left(\omega_{0}\right)\left[n_{p h}\left(\omega_{0}\right)+1\right] .
\end{aligned}
$$

We Fourier transform this system with respect to charge and energy,

$$
\begin{aligned}
& \mathcal{P}_{t}\left(n, \chi, \eta_{L}, \eta_{R}, \xi\right)= \\
& \sum_{N=-\infty}^{\infty} e^{i N \chi} \sum_{q=-\infty}^{\infty} e^{i q \omega_{0} \xi} \int_{-\infty}^{\infty} e^{i \omega_{L} \eta_{L}} d \omega_{L} \\
& \times \int_{-\infty}^{\infty} e^{i \omega_{R} \eta_{R}} d \omega_{R} \mathcal{P}_{t}\left(n, N, \omega_{L}, \omega_{R}, q \omega_{0}\right)
\end{aligned}
$$

to obtain the characteristic function $\mathcal{Z}\left(\chi, \eta_{L}, \eta_{R}, \xi, t\right)$. It depends on the energy counting fields $\eta_{\nu}$ and $\xi$, and the charge counting field $\chi$. It is a vector with two entries, as in Eq. (28), and it satisfies the differential equation

$$
\frac{d|\mathcal{Z}\rangle}{d t}=-\hat{\mathcal{W}}|\mathcal{Z}\rangle
$$

The matrix $\hat{\mathcal{W}}$ has the following entries

$$
\begin{aligned}
& \hat{\mathcal{W}}= \\
& \left(\begin{array}{cc}
k_{0 \rightarrow 1}^{e}+\Gamma_{p h} n_{p h} & -e^{i \chi} F_{1}^{-}\left(\eta_{L}, \eta_{R}\right)-e^{-i \chi} F_{2}^{+}\left(\eta_{L}, \eta_{R}\right)-\Gamma_{p h}\left(n_{p h}+1\right) e^{i \xi \omega_{0}} \\
-e^{i \chi} F_{1}^{+}\left(\eta_{L}, \eta_{R}\right)-e^{-i \chi} F_{2}^{-}\left(\eta_{L}, \eta_{R}\right)-\Gamma_{p h} n_{p h} e^{-i \xi \omega_{0}} & k_{1 \rightarrow 0}^{e}+\Gamma_{p h}\left(n_{p h}+1\right)
\end{array}\right)
\end{aligned}
$$

where

$$
\begin{aligned}
F_{1}^{ \pm}\left(\eta_{L}, \eta_{R}\right) & =\int_{-\infty}^{\infty} e^{-i \epsilon \eta_{L}} e^{i\left(\epsilon \mp \omega_{0}\right) \eta_{R}} \\
& \times f_{L}(\epsilon)\left[1-f_{R}\left(\epsilon \mp \omega_{0}\right)\right] J_{L}(\epsilon) J_{R}\left(\epsilon \mp \omega_{0}\right) d \epsilon
\end{aligned}
$$

and

$$
\begin{aligned}
F_{2}^{ \pm}\left(\eta_{L}, \eta_{R}\right) & =\int_{-\infty}^{\infty} e^{i \eta_{L}\left(\epsilon \pm \omega_{0}\right)} e^{-i \epsilon \eta_{R}} \\
& \times\left[1-f_{L}\left(\epsilon \pm \omega_{0}\right)\right] f_{R}(\epsilon) J_{L}\left(\epsilon \pm \omega_{0}\right) J_{R}(\epsilon) d \epsilon
\end{aligned}
$$


The CGF is expressed in terms of the characteristic function $|\mathcal{Z}\rangle$ as

$$
G\left(\chi, \eta_{L}, \eta_{R}, \xi\right)=\lim _{t \rightarrow \infty} \frac{1}{t} \ln \langle I \mid \mathcal{Z}\rangle,
$$

Practically, it is reached by the negative of the smallest eigenvalue of the matrix $\hat{\mathcal{W}}$,

$$
\begin{aligned}
G\left(\chi, \eta_{L}, \eta_{R}, \xi\right) & =-\frac{w_{1,1}+w_{2,1}}{2} \\
& +\frac{\sqrt{\left(w_{1,1}-w_{2,2}\right)^{2}+4 w_{1,2} w_{2,1}}}{2}
\end{aligned}
$$

with $w_{i, j}$ the matrix elements in Eq. (A9). The charge current is obtained by taking the first derivative with respect to $(i \chi)$,

$$
\begin{aligned}
\left\langle I_{e}\right\rangle & =\left.\frac{\partial G}{\partial i \chi}\right|_{\chi, \eta_{\nu}, \xi=0}=\left(w_{1,1}+w_{2,2}\right)^{-1} \\
& \times\left.\left[w_{2,1} \frac{\partial w_{1,2}}{\partial i \chi}+w_{1,2} \frac{\partial w_{2,1}}{\partial i \chi}\right]\right|_{\chi, \eta_{\nu, \xi=0}},
\end{aligned}
$$

where the following holds when the counting fields are all set to zero, $w_{1,2}=-w_{2,2}, w_{2,1}=-w_{1,1}, \partial w_{1,2} /\left.\partial(i \chi)\right|_{0}=$ $k_{1 \rightarrow 0}^{R \rightarrow L}-k_{1 \rightarrow 0}^{L \rightarrow R}$ and $\partial w_{2,1} /\left.\partial(i \chi)\right|_{0}=k_{0 \rightarrow 1}^{R \rightarrow L}-k_{0 \rightarrow 1}^{L \rightarrow R}$. Recall that the rates $k_{n \rightarrow n^{\prime}}^{\nu \rightarrow \nu^{\prime}}$ are electron-hole generation assisted rates, see Eq. (25). We can now identify the levels population,

$$
\begin{aligned}
\frac{w_{1,1}}{w_{1,1}+w_{2,2}} & =\frac{k_{0 \rightarrow 1}^{e}+\Gamma_{p h} n_{p h}}{k_{0 \rightarrow 1}^{e}+k_{1 \rightarrow 0}^{e}+\Gamma_{p h}\left[2 n_{p h}+1\right]} \\
& =p_{1},
\end{aligned}
$$

and similarly for $p_{0}=1-p_{1}$, resulting in the expression for the charge current

$$
\left\langle I_{e}\right\rangle=p_{1}\left(k_{1 \rightarrow 0}^{L \rightarrow R}-k_{1 \rightarrow 0}^{R \rightarrow L}\right)+p_{0}\left(k_{0 \rightarrow 1}^{L \rightarrow R}-k_{0 \rightarrow 1}^{R \rightarrow L}\right) .(
$$

This result is formally identical to Eq. (48), with the only difference that the steady-state TLS population is now modified, to include phonon-bath assisted transitions.
[1] L. H. Yu., Z. K. Keane, J. W. Ciszek, L. Cheng, M. P. Stewart, J. M. Tour, and D. Natelson, Phys. Rev. Lett. 93, 266802 (2004).

[2] D. Djukic, K. S. Thygesen, C. Untiedt, R. H. M. Smit, K.W. Jacobsen, and J. M. van Ruitenbeek, Phys. Rev. B 71, 161402 (2005).

[3] A. N. Pasupathy, J. Park, C. Chang, A. V. Soldatov, S. Lebedkin, R. C. Bialczak, J. E. Grose, L. A. K. Donev, J. P. Sethna, D. C. Ralph, and P. L. McEuen, Nano Lett. 5, 203 (2005).

[4] Z. Ioffe, T. Shamai, A. Ophir, G. Noy, I. Yutsis, K. Kfir, O. Cheshnovsky, and Y. Selzer, Nature Nanotech. 3, 727 (2008) .

[5] D. R. Ward, D. A. Corley, J. M. Tour, and D. Natelson, Nature Nanotech. 6, 33 (2011).

[6] D. Segal and A. Nitzan, J. Chem. Phys. 117, 3915 (2002).

[7] Y.-C. Chen, M. Zwolak, M. Di Ventra, Nano Lett. 3, 1691 (2005).

[8] Z. Huang, F. Chen, R. Dagosta, P. A. Bennett, M. Di. Ventra, N. Tao, Nature Nanotech. 2, 698 (2007).

[9] M. Galperin, M. A. Ratner, and A. Nitzan, J. Phys.: Condens. Matter 19, 103201 (2007), and references therein.

[10] R. Härtle and M. Thoss, Phys. Rev. B 83, 125419 (2011).

[11] F. Giazotto, T. T. Heikkila, A. M. Savin, and J. P. Pekola, Rev. Mod. Phys. 78, 217 (2006).

[12] M. Galperin, K. Saito, A. V. Batalsky, and A. Nitzan, Phys. Rev. B 80, 115427 (2009).

[13] R. Härtle and M. Thoss, Phys. Rev. B 83, 115414 (2011).

[14] A. Aviram and M. A. Ratner, Chem. Phys. Lett. 29, 277 (1974).

[15] I. Diez-Perez, J. Hihath, Y. Lee, L. Yu, L. Adamska, M. A. Kozhushner, I. I. Oleynik, and N. Tao, Nature Chem. 1, 635 (2009).

[16] J.-T. Lü, P. Hedegard, and M. Brandbyge, Phys. Rev.
Lett. 107, 046801 (2011).

[17] R. Volkovich, R. Härtle, and M. Thoss, Phys. Chem. Chem. Phys. 32, 14333 (2010).

[18] R. Jorn and T. Seideman, Acc. Chem. Res. 43, 1186 (2010).

[19] D. J. Evans, E. G. D. Cohen, and G. P. Morriss, Phys. Rev. Lett. 71, 2401 (1993).

[20] G. Gallavotti and E. G. D. Cohen, Phys. Rev. Lett. 74, 2694 (1995).

[21] G. M. Wang, E. M. Sevick, E. Mittag, D. J. Searles, and D. J. Evans, Phys. Rev. Lett. 89, 050601 (2002).

[22] S. Nakamura, Y. Yamauchi, M. Hashisaka, K. Chida, K. Kobayashi, T. Ono, R. Leturcq, K. Ensslin, K. Saito, Y. Utsumi, and A. C. Gossard, Phys. Rev. Lett. 104, 080602 (2010); Phys. Rev. B 83, 155431 (2011).

[23] M. Esposito, U. Harbola, and S. Mukamel, Rev. Mod. Phys. 81, 1665 (2009).

[24] M. Campisi, P. Hänggi, and P. Talkner, Rev. Mod. Phys. 83, 771 (2011).

[25] D. Andrieux, P. Gaspard, T. Monnai, and S. Tasaki, New J. Phys. 11, 043014 (2009).

[26] L. Nicolin and D. Segal, Phys. Rev. B 84, 161414 (2011).

[27] L. Nicolin and D. Segal, J. Chem. Phys. 135, 164106 (2011).

[28] C. Emary, J. Phys. Condens. Matter 23, 025304 (2011).

[29] A. Braggio, J. Koenig and R. Fazio, Phys. Rev. Lett. 96, 026805 (2006); C. Flindt, T. Novotny, A. Braggio, M. Sassetti and A.-P. Jauho, Phys. Rev. Lett. 100, 150601 (2008).

[30] J. Bonca and S. A. Trugman, Phys. Rev. Lett. 79, 4874 (1997).

[31] M. Galperin, A. Nitzan, and M. A. Ratner, Phys. Rev. B 75, 155312 (2007); M. Galperin, M. A. Ratner, and A. Nitzan, J. Chem. Phys. 130, 144109 (2009).

[32] A. Mitra, I. Aleiner, and A. J. Millis, Phys. Rev. B 69, 
245302 (2004).

[33] T. Novotny, A. Donarini, C. Flindt, and A.-P. Jauho, Phys. Rev. Lett. 92, 248302 (2004).

[34] Y. Asai, Phys. Rev. B 78, 045434 (2008).

[35] T.-H. Park and M. Galperin, Phys. Rev. B 84, 205450 (2011).

[36] S. Maier, T. L. Schmidt, and A. Komnik, Phys. Rev. B 83, 085401 (2011).

[37] G. D. Mahan, Many-particle physics (Plenum press, New York, 2000).

[38] D. Segal, Phys. Rev. B 73, 205415 (2006).

[39] T. Krause, G. Schaller, and T. Brandes, Phys. Rev. B 84, 195113 (2011).

[40] A. Mitra and A. J. Millis, Phys. Rev. B 72, 121102(R) (2005); Phys. Rev. B 76, 085342 (2007).

[41] D. Segal, D. R. Reichman, and A. J. Millis, Phys. Rev. B 76, 195316 (2007).

[42] D. Segal, A. J. Millis, and D. R. Reichman, Phys. Rev. B 82, 205323 (2010).

[43] H.-P. Breuer and F. Petruccione, The Theory of Open Quantum Systems Oxford University Press, New York,
New York, (2002).

[44] D. R. Reichman and R. J. Silbey, J. Chem. Phys. 104, 1506 (1996).

[45] V. Mujica, A. E. Roitberg, and M. A. Ratner, J. Chem. Phys. 112, 6834 (2000).

[46] U. Harbola, M. Esposito, and S. Mukamel, Phys. Rev. B 76, 085408 (2007).

[47] J. Ren, P. Hänggi, and B. Li, Phys. Rev. Lett. 104, 170601 (2010).

[48] D. R. Schmidt, R. J. Schoelkopf, and A. N. Cleland, Phys. Rev. Lett. 93, 045901 (2004).

[49] M . Meschke, W. Guichard, and J. P. Pekola, Nature 444, 187 (2006).

[50] J. P. Pekola, V. F. Maisi, S. Kafanov, N. Chekurov, A. Kemppinen, Yu. A. Pashkin, O.-P. Saira, M. Mttnen, and J. S. Tsai, Phys. Rev. Lett. 105, 026803 (2010)

[51] T. Ojanen and T. T. Heikkila, Phys. Rev. B 76, 073414 (2007); T. Ojanen and A.-P Jauho, Phys. Rev. Lett. 100, 155902 (2008).

[52] D. Segal, Phys. Rev. Lett. 100, 105901 (2008). 